\title{
THE EXISTENCE OF INVARIANT EINSTEIN METRICS ON A COMPACT HOMOGENEOUS SPACE
}

\author{
M. M. GRAEV
}

\begin{abstract}
It is proved that if the triangularizable compact set $C=X_{G, H}^{\Sigma}$ introduced by Böhm (Böhm's polyhedron) is non-contractible, then there exists a positivedefinite invariant Einstein metric $m$ of positive scalar curvature on a connected homogeneous space $G / H$ of a compact Lie group $G$. There is a natural continuous map of $C$ onto the flag complex $K_{B}$ of a finite graph $B$. For $C=K_{B}$ this gives one of the criteria proved by Böhm. Another consequence - that $m$ exists for disconnected $B$ - is a version of the Böhm-Wang-Ziller graph theorem (but now the graph may be disconnected for $\mathfrak{z}(\mathfrak{g}) \neq 0$ ). Furthermore, the preparatory Böhm theorems on retractions are revised, and in this connection new constructions of certain topological spaces are proposed.
\end{abstract}

\section{$\S$ 1. Introduction. BÖHM's EXISTEnCE CRITERION AND A GENERALIZATION}

This paper is devoted to a criterion for invariant Einstein metrics $g: \operatorname{ric}(g)=\lambda g$ to exist on a compact homogeneous manifold.

In what follows we consider a connected $n$-dimensional homogeneous space $G / H$ of a compact Lie group $G$. We denote the complete Riemannian manifold of all $G$-invariant Riemannian metrics of volume 1 on $G / H$ by $\mathcal{M}_{1}^{G}$ (it is diffeomorphic to $\mathbb{R}^{N}$ for $N<$ $\left.\frac{n(n+1)}{2}\right)$. Recall that the Einstein metrics belonging to $\mathcal{M}_{1}^{G}$ coincide with the critical points of the smooth function $g \in \mathcal{M}_{1}^{G} \mapsto \operatorname{sc}(g) \in \mathbb{R}$ (the scalar curvature of a metric $g$ ). Furthermore, if $G / H$ is different from a torus, then all the critical values of $\operatorname{sc}(g)$ are strictly positive. (See also $\S 2$ )

Several years ago Böhm proved that if a compact polyhedron, which he constructed, is non-contractible, then invariant positive-definite Einstein metrics exist on a compact connected homogeneous space $G / H$.

In the Introduction we describe Böhm's polyhedron explicitly, state Böhm's criterion and a generalization of it. To do this we need the following definitions.

On the group $G$ we fix a bi-invariant Riemannian metric $Q$ and also denote its projection onto $G / H$ by $Q$. We normalize $Q$ in such a way that $Q \in \mathcal{M}_{1}^{G}$. Let $\mathcal{S}$ denote the topological space of geodesic rays on $\mathcal{M}_{1}^{G}$ going out from the point $Q$; this space has a natural identification with the unit sphere $\Sigma$ in $T_{Q} \mathcal{M}_{1}^{G}$, but we shall also use other models.

Let $\mathcal{S}_{k} \subset \mathcal{S}$ be the subset of rays intersecting the levels $\operatorname{sc}(g) \geq k$.

Böhm constructed a compact subspace $X_{\mathcal{E}} \subset \mathcal{S}$ with the following properties:

(a) every neighbourhood of the subspace $X_{\varepsilon}$ contains $\mathcal{S}_{k}$ for $k \gg 0$;

(b) $X_{\varepsilon}$ is a neighbourhood retract of the sphere $\mathcal{S}$;

(c) $X_{\varepsilon}$ contracts over itself to the polyhedron $\|\mathcal{K}\|$, which is described explicitly below. The polyhedron $\|\mathcal{K}\| \subset X_{\varepsilon}$ satisfies the following condition:

2010 Mathematics Subject Classification. Primary 53C25; Secondary 53C30.

Key words and phrases. Homogeneous manifold, Einstein metric.

This research was supported by the Russian Foundation for Basic Research (grant no. 10-01-00041a). 
(d) for every $t \geq 0$ and every ray $r \in\|\mathcal{K}\|$ the inequality $\operatorname{sc}(r(t))>c e^{t / n}>0$ holds, where $c$ is a constant independent of $r$ and $t$, and $t \mapsto r(t)$ is the natural parametrization.

Böhm's criterion can be derived from (a)-(d) and the variational theorem obtained in BWZ by a certain argument; see $B$ and $\S 2$ below.

Moreover, Böhm proved the following theorem:

(e) the intersections of the levels $\operatorname{sc}(g)>2 \mathrm{sc}(Q)$ by every sufficiently remote sphere $\{r(t): r \in \mathcal{S}\}, t \gg 0$, identified with $\mathcal{S}$ are contained in a pre-assigned neighbourhood of $X_{\varepsilon}$. This theorem obviously implies (a).

We point out that the third property of $X_{\varepsilon}$ was proved in $[\mathrm{B}$. only under additional restrictions 11 on the homogeneous space $G / H$.

In this paper we get rid of all the restrictions on $G / H$, but to do this we have to sacrifice the natural Böhm triangulation on $\|\mathcal{K}\|$, which does not exist in the general case, and consider $\|\mathcal{K}\|$ merely as a triangularizable compact set. Furthermore, we propose a new and simpler construction of a space $X_{\varepsilon}$ with properties (a)-(c) and (e).

In the case considered by Böhm it is convenient to introduce the polyhedron $\|\mathcal{K}\|$ algebraically, that is, as a simplicial scheme; its vertices are subalgebras of the Lie algebra $\mathfrak{g}$ in a certain finite set $\mathcal{K}$, and the simplexes are all possible flags $\varphi$ of subalgebras in this set.

In order to obtain a geometric realization of the complex $\Delta(\mathcal{K})$ of flags $\varphi$, we firstly introduce an $\operatorname{Ad}(G)$-invariant Euclidean metric on the Lie algebra $\mathfrak{g}$ and associate with every subalgebra $\mathfrak{k} \in \mathcal{K}$ the orthoprojector $\chi^{\mathfrak{k}}=1_{\mathfrak{g}}-1_{\mathfrak{k}}$. Now, with every flag $\varphi \in \Delta(\mathcal{K})$ we can associate the rectilinear simplex $\|\varphi\|$, that is, the convex hull of all the $\chi^{\mathfrak{f}}, \mathfrak{f} \in \varphi$.

Let $\|\mathcal{K}\|$ denote the union of simplexes $\|\varphi\|, \varphi \in \Delta(\mathcal{K})$. Points $A \in\|\mathcal{K}\|$ are symmetric operators with non-negative spectrum on $\mathfrak{g}$ and lie on the hypersurface of operators with largest eigenvalue equal to one 2

Böhm considered the following families of subalgebras $\mathcal{K}$ as candidates:

1) all $\operatorname{Ad}(H)$-invariant proper subalgebras $\mathfrak{k} \subset \mathfrak{g}$ properly containing $\mathfrak{h}$ such that $[\mathfrak{k}, \mathfrak{k}] \not \subset \mathfrak{h}$ (non-toral $H$-subalgebras in his terminology);

2 ) the subalgebras spanned by finite sums of minimal subalgebras $\mathfrak{k}$ satisfying condition $1)$;

3) and 4) are defined in the same way as 1) and 2), respectively, but the condition of $\operatorname{Ad}(H)$-invariance is supplemented by the invariance under the adjoint action of a maximal torus $T$ of the group $\operatorname{Norm}_{G^{0}}\left(H^{0}\right)$.

It is possible to consider other families in a similar way, in particular,

$1^{*}$ ) the subalgebras $\mathfrak{l}$ of type 1 ) satisfying the condition $\mathfrak{l}=[\mathfrak{l}, \mathfrak{l}]+\mathfrak{h}$, which we call almost semisimple.

We point out that the subalgebra spanned by every pair of almost semisimple subalgebras $1^{*}$ ) is almost semisimple or is equal to $\mathfrak{g}$, every subalgebra 1 ) contains an almost semisimple subalgebra, and every subalgebra 2 ) is almost semisimple.

\footnotetext{
${ }^{1}$ In more detail, Böhm introduced the auxiliary "space of non-toral directions" $X_{n t}^{\Sigma}$ and proved the existence of strong deformation retractions $X_{\varepsilon} \stackrel{1}{\rightarrow} X_{n t}^{\Sigma} \stackrel{2}{\rightarrow}\|\mathcal{K}\|$. For 2 he used a special triangulation $\tau$ on the polyhedron $\|\mathcal{K}\|$ (in the main case, denoted by $X_{G / H}^{\Sigma}$ and called the "nerve" [B] $\left.\S 1\right]$ ) and conjectured, if I understand correctly, that it is possible to do without $\tau$.

${ }^{2}$ This hypersurface is inscribed into the Euclidean sphere $\left\{A: d\left(A, \frac{1}{2} 1_{\mathfrak{g}}\right)=\frac{1}{2} \sqrt{\operatorname{dim} \mathfrak{g}}\right\}$, which contains vertices of the complex, and we can speak about the stereographical projection onto a hyperplane from the pole 0 . Below, in the main body of the paper, we use the second rectilinear model obtained from the first model by a similar stereographical projection. In that model every Euclidean triangle $\mathfrak{k}_{1}>\mathfrak{k}_{2}>\mathfrak{k}_{3}$, $\mathfrak{k}_{i} \in \mathcal{K}$, has a right angle at the vertex $\mathfrak{k}_{2}$. Böhm used the third, spherical, model of the complex, where every edge is strictly less than a quarter of the great circle on which it lies, and every Euler triangle $\mathfrak{k}_{1}>\mathfrak{k}_{2}>\mathfrak{k}_{3}$ again has a right angle at the vertex $\mathfrak{k}_{2}$.
} 
By Proposition 4.2 in [BWZ, the family $1^{*}$ ) consists of finitely many orbits of the connected component of the identity of the compact group $\operatorname{Norm}_{G}(H)$.

Böhm proved that the set 4) is finite. The families of subalgebras 1)-3) may turn out to be finite (in particular, empty) or to have the cardinality of the continuum.

Böhm's criterion (See [B] $)$. Let $\mathcal{K}$ be one of the sets 1$)-4)$ or $\left.1^{*}\right)$. Suppose that $\mathcal{K}$ is finite, and the corresponding simplicial scheme $\Delta(\mathcal{K})$ is non-contractible (for example, empty). Then there exists a positive-definite Einstein metric on $G / H$ that is invariant under the action of the group $G$ and, in cases 3) and 4), also under the right action of the torus $T$.

A related criterion was obtained in the Böhm-Wang-Ziller paper [BWZ]. According to [BWZ, the existence of such Einstein metrics follows from the fact that a certain compact topological space $Y_{\mathrm{WZ}} \subset \mathcal{S}$ is disconnected, which in turn follows because a certain finite graph $\Upsilon_{\mathrm{WZ}}$ is disconnected (Graph Theorem 3 ).

Instead of $Y_{\mathrm{WZ}}$, the proof of the criterion in [B] uses a smaller topological space, $X_{\varepsilon}$, which, as was shown there, contracts over itself to a compact polyhedron - the finite simplicial Böhm complex and, finally, the existence of invariant Einstein metrics on $G / H$ follows from the non-contractibility of this simplicial complex 4 Note that in cases 3 ) and 4) the manifold of metrics $\mathcal{M}_{1}^{G}$ is replaced by the totally geodesic submanifold of $T$-invariant metrics $\left(\mathcal{M}_{1}^{G}\right)^{T}$ and the corresponding changes are made in the definition of $X_{\varepsilon}$.

In $\S 5.6$ of $\left[\mathrm{B}\right.$ the construction of $X_{\varepsilon}$ alone, even before properties (a) and (c) are verified, includes an inductive definition and several pages of preparation. This construction looks cumbersome by comparison with the construction of the space $Y_{\mathrm{WZ}}$ in $\S 3$ of BWZ; the latter consists of linear connected components of a larger space, which is described in what follows with minor differences from the original (see Remark 4.3).

In this paper we put forward a different construction of $X_{\varepsilon}$. This space is contained between the same closed semi-algebraic subsets $X_{n t}^{\Sigma}$ and $W^{\Sigma}$ of the sphere $\mathcal{S} \simeq \Sigma$ as in [B] (sometimes, $X_{n t}^{\Sigma}=X_{\varepsilon}=W^{\Sigma}$, for example, when $\operatorname{rank}(G)=\operatorname{rank}(H)$ ).

It is possible to describe the behaviour of the scalar curvature at infinity by the same Böhm formulae, where $\|\mathcal{K}\| \subset X_{\varepsilon} \subset W^{\Sigma} \subset \Sigma \simeq \mathcal{S}$ :

$$
\lim _{t \rightarrow+\infty} \operatorname{sc}(r(t)) \begin{cases}=+\infty, & r \in\|\mathcal{K}\|, \\ \leq 0, & r \in W^{\Sigma} \backslash X_{\varepsilon}, \\ =-\infty, & r \in \Sigma \backslash W^{\Sigma} .\end{cases}
$$

In the new construction of the space $X_{\varepsilon}$ we also use a compact set $\|\mathcal{T}\|$ whose role was not noticed in [B]. Let $\mathcal{T}$ denote the family of toral $H$-subalgebras $\mathfrak{k}$ which is defined in the same way as the family 1 ), with the condition $[\mathfrak{k}, \mathfrak{k}] \not \subset \mathfrak{h}$ replaced by $[\mathfrak{k}, \mathfrak{k}] \subset \mathfrak{h}$. Every toral subalgebra splits into a direct sum of the subalgebra $\mathfrak{h}$ and a non-zero Abelian subalgebra. Note that the set $\mathcal{T}$, generally speaking, has the cardinality of the continuum. By analogy with $\|\mathcal{K}\|$, we define the subset of symmetric linear operators $\|\mathcal{T}\|$. This is a compact

\footnotetext{
3 "This theorem can be viewed as another step towards a general understanding of the existence and non-existence of homogeneous Einstein metrics, and was suggested by the last two authors 15 years ago" BWZ].

${ }^{4}$ In fact, this theorem was obtained there in several versions with differing restrictions on their applicability. One of them can be applied to an arbitrary compact homogeneous space $G / H$ and $G$-invariant metrics that are invariant also under the right action of a maximal torus of the group $\operatorname{Norm}_{G^{0}}\left(H^{0}\right)$. Under this restriction on the metrics, Böhm's complex is finite (it depends on the space of metrics), and when this restriction is discarded, the finiteness of the complex becomes a restriction on $G / H$. See also BK] and GLP] concerning applications.
} 
semi-algebraic subset of the Euclidean space $\mathfrak{g l}(\mathfrak{g})$, which has empty intersection with $\|\mathcal{K}\|$, that is, $\|\mathcal{K}\| \cap\|\mathcal{T}\|=\varnothing$.

The following theorems are new.

Theorem A. There exists a compact subspace $X_{\varepsilon} \subset W^{\Sigma}$, with properties (a), (b), that can be embedded into the join $J=\|\mathcal{T}\| * X_{n t}^{\Sigma}$ in such a way that the natural contraction of the complement $J \backslash\|\mathcal{T}\|$ to the second factor induces a strong deformation retraction of $X_{\varepsilon}$ to $X_{n t}^{\Sigma}$.

By construction, $X_{\varepsilon}$ will be a semi-algebraic set (as in [B]). This implies (b).

By $[\mathrm{B}$, Theorems 5.52 and 5.54, property (a) holds if

(*) $W^{\Sigma} \backslash X_{\varepsilon}$ is covered by the two subsets defined in Corollary 5.49 in [B] by formulae (5.50) and (5.51).

Therefore below, instead of property (a), we verify this geometric condition 5 which we write out directly in a form convenient to us.

Theorem $\mathrm{A}$ follows from Propositions 6.2 and 6.3 below (Corollary 6.4).

The compact set $X_{\varepsilon}$ constructed above, or any other semi-algebraic compact set lying on $W^{\Sigma}$, contractible over itself to $X_{n t}^{\Sigma}$, and satisfying condition $(*)$, is called a suitable extension of the space of non-toral directions $X_{n t}^{\Sigma}$, which does not contradict Böhm's terminology.

Böhm proved that $X_{n t}^{\Sigma}$ contracts over itself to $\|\mathcal{K}\|$, where $\mathcal{K}$ is the family 1) or 2), under the assumption that $\mathcal{K}$ is finite. In this paper we prove a similar assertion (which can be applied to many families) for compact $\mathcal{K}$ and $\|\mathcal{K}\|$.

Suppose for definiteness that $\mathcal{K}$ is a family of subalgebras of type 1 ), $1^{*}$ ), or 2 ). Suppose that the union $\|\mathcal{K}\|$ of simplexes $\|\varphi\|, \varphi \in \Delta(\mathcal{K})$, is a compact subset of the Euclidean space $\mathfrak{g l}(\mathfrak{g})$. Then the following holds.

Theorem B. If the space $\|\mathcal{K}\|$ with topology inherited from $\mathfrak{g l}(\mathfrak{g})$, is compact, then it is a strong deformation retract of the spaces $X_{\varepsilon}$ and $X_{1}=X_{n t}^{\Sigma}$.

We point out that in the general case $\|\mathcal{K}\|$ being compact is equivalent to the compactness of $\mathcal{K} \simeq\left\{A \in\|\mathcal{K}\|: d\left(A, \frac{1}{2} 1_{\mathfrak{g}}\right)=\frac{1}{2} \sqrt{\operatorname{dim} \mathfrak{g}}\right\}$. (Here, $d(u, v)$ is the Euclidean distance on $\mathfrak{g l}(\mathfrak{g})$.)

Theorem $\mathrm{B}$ follows from Theorem 6.6 below in view of the remark above and Theorem $\mathrm{A}$ on existence.

One can prove that families $\mathcal{K}$ of types 1$\left.), 1^{*}\right), 2$ ) are compact, and Theorem $B$ can be applied to them. For example, in case $\left.1^{*}\right)$ compactness follows because the quotient $\mathcal{K} / \operatorname{Norm}_{G}(H)^{0}$ is finite. For the same reason, the family 2) consists of connected components of the family $1^{*}$ ) and so is compact. (With an appropriate definition of $X_{\varepsilon}$ and $X_{n t}^{\Sigma}$, Theorem $\mathrm{B}$ also admits a generalization to a family $\mathcal{K}$ of type 3 ), whose compactness follows easily from the compactness of 1 ), and to many other families of subalgebras.)

In case 2$)$, there is a homeomorphism of $\|\mathcal{K}\|$ onto the topological space $X_{G / H}^{\Sigma}$ in $\mathrm{B}$ (called the nerve). As is clear from the definition, the nerve has a fairly nice structure. Böhm calls it a semi-algebraic variety. In $[\mathrm{B}, \S 1$, Böhm stated without proof that the nerve is compact, and we have already discussed this. Then by Theorem $\mathrm{B}$ the conjecture stated there, that $X_{G / H}^{\Sigma}$ is a strong deformation retract of the space $X_{n t}^{\Sigma}$, is true in the general case (it was proved in $[\mathrm{B}]$ only for finite $\mathcal{K}$ ).

\footnotetext{
${ }^{5}$ Böhm proved that under this condition for every $\delta$-neighbourhood $U_{\delta}$ of the compact set $X_{\varepsilon}$ in $\mathcal{S}$ there exists a number $t_{0}(\delta)>0$ such that $\operatorname{sc}(r(t)) \leq 2 \operatorname{sc}(Q)$ for all $t>t_{0}(\delta)$ and all $r \in \mathcal{S} \backslash U_{\delta}$.
} 
Now, both in Böhm's criterion and in its proof (see [B], Theorems 1.4 and 8.1), we can replace the non-contractibility of the finite simplicial scheme $\Delta(\mathcal{K})$ by the noncontractibility of the compact set $\|\mathcal{K}\| \subset \mathfrak{g l}(\mathfrak{g})$. This is possible by Theorems $\mathrm{A}$ and B and the proof (which is in fact based on properties (a)-(d)) does not need to be revised.

Thus, by passing from finite $\mathcal{K}$ to compact $\mathcal{K}$ and combining Theorems $\mathrm{A}$ and $\mathrm{B}$ with Böhm's concluding argument, we obtain the following theorem.

Theorem C. If $\|\mathcal{K}\| \subset \mathfrak{g l}(\mathfrak{g})$ is a non-contractible compact set, then on the manifold $G / H$ there exists an invariant positive-definite Einstein metric.

It is well known that such metrics do not exist at all if the compact homogeneous manifold $G / H$ is different from a torus and has infinite-fundamental group. In this case, $\mathfrak{h}<[\mathfrak{g}, \mathfrak{g}]+\mathfrak{h}<\mathfrak{g}$, that is, there exists a largest subalgebra $\mathfrak{k}$ of type $1^{*}$ ), and then also a largest subalgebra $\mathfrak{k}=\mathfrak{k}_{\max }$ of type 2 ). The definition immediately implies that $\|\mathcal{K}\| \simeq X_{G / H}^{\Sigma}$ contracts over itself to the point $\chi^{\mathfrak{k}_{\max }}$ (exactly as in the proof of Proposition 7.5 in $[\mathrm{B}]$ ), and no contradiction arises.

Below in $\S 2$ we construct a family of homogeneous spaces $G / H$ on which there exist invariant Einstein metrics by Theorem C, although neither Böhm's existence criterion nor the Graph Theorem make it possible to assert anything. To do this we derive a new version of the Graph Theorem from Theorem C.

Let $\mathcal{L}$ be the family of all almost semisimple subalgebras of type $\left.1^{*}\right)$, and $\mathcal{L}^{\text {min }} \subset \mathcal{L}$ the subfamily of subalgebras of type 2). Let $[\mathfrak{l}]$ denote the orbit of every subalgebra $\mathfrak{l} \in \mathcal{L}$ with respect to $\operatorname{Norm}_{G}(H)^{0}$. Let $\mathrm{B}_{\mathrm{WZ}}$ denote the graph with vertices $[\mathfrak{l}], \mathfrak{l} \in \mathcal{L}$, and edges $([\mathfrak{k}],[\mathfrak{l}])$, where $\mathfrak{k}<\mathfrak{l}$. Let $B_{\mathrm{WZ}}^{\min }$ be the subgraph of this graph induced on the subset of vertices $[\mathfrak{k}], \mathfrak{k} \in \mathcal{L}^{\text {min }}$. Then

$$
\mathrm{B}_{\mathrm{WZ}}^{\min } \subset \mathrm{B}_{\mathrm{WZ}} \subset \Upsilon_{\mathrm{WZ}}
$$

These three graphs are finite. There exist continuous surjective maps of the compact sets $\|\mathcal{K}\|=\|\mathcal{L}\|$ and $\left\|\mathcal{L}^{\min }\right\|$ onto the flag complexes of the graphs $\mathrm{B}_{\mathrm{WZ}}$ and $\mathrm{B}_{\mathrm{WZ}}^{\min }$, respectively. Hence Theorem $\mathrm{C}$ implies the following.

Corollary (A variant Graph Theorem). If the graph $\mathrm{B}_{\mathrm{WZ}}$ or $\mathrm{B}_{\mathrm{WZ}}^{\min }$ is disconnected, there exists an invariant Einstein metric on $G / H$.

By Theorem $\left[\right.$, if $\|\mathcal{L}\|$ or $\left\|\mathcal{L}^{\text {min }}\right\|$ is non-contractible, invariant Einstein metrics exist simultaneously on $G / H$ and $\bar{G} / \bar{H}$, where $\bar{G}=G / Z$ is the quotient of $G$ by some closed connected commutative normal subgroup, and $\bar{H}=H / H \cap Z$. Suppose, for example, that

$$
\bar{G} / \bar{H}=\left(G_{1} / H_{1} \times \ldots \times G_{p} / H_{p}\right) / Z,
$$

where $G_{1} / H_{1}$ is a simply connected homogeneous Einstein space with disconnected graphs $\mathrm{B}_{\mathrm{WZ}}$ and $\mathrm{B}_{\mathrm{WZ}}^{\min }$, and every quotient $G_{i} / H_{i}$ with $i>1$ is a principal bundle of circles over an irreducible Hermitian symmetric space and has the natural Sasaki-Einstein geometry with full isometry group $G_{i}$. Then on $\bar{G} / \bar{H}$ there exists an invariant Einstein metric (see $\S 2$ ).

This paper contains more than just Theorems $\mathrm{A}$, B, and C] and these are stated for special cases. The first of them clarifies the paper $[\mathrm{B}$; the second, roughly speaking, asserts the validity of one its conjectures; and the third follows from the first two and contains a certain generalization of the criterion. One additional result in the paper is a simple construction of $X_{\varepsilon}$. Besides this, the paper contains a revision of Böhm's preparatory Theorems 5.48 and 6.10 on retractions of subspaces of the topological space $W^{\Sigma}$.

"Rough draft" versions of these theorems are also obtained, in which $W^{\Sigma}$ and $X_{\varepsilon}$ are replaced by larger spaces. To obtain the rough versions, the starshaped semi-algebraic 
sets appearing in Böhm's constructions are replaced by balls. The rough draft version of $W^{\Sigma}$ is different from its rough version in BWZ (see below, Remark 4.3); in particular, it is independent of the parameters. To prove Böhm's criterion and Theorem C, it is sufficient to use the rough draft version of $X_{\varepsilon}$ (with the requisite properties (a)-(c)).

Starting from $\S 5$ we take elementary contractible subspaces for a basis; we call these butterflies. An intersection of butterflies is again a butterfly. The definitive formula (5.2) is found for the intersection; the main special cases of this were in fact obtained by Böhm:

$\mathrm{B}\left[\varphi_{1}\right] \cap \mathrm{B}\left[\varphi_{2}\right]=\mathrm{B}\left[\varphi_{1} \varphi_{2}\right]$, where $\mathrm{B}[\varphi]$ is the butterfly associated with every flag of subalgebras $\varphi$, and $\left(\varphi_{1}, \varphi_{2}\right) \mapsto \varphi=\varphi_{1} \varphi_{2}$ is a commutative associative idempotent multiplication of flags.

For example, the butterflies associated with flags of the form $\varphi=\left(\mathfrak{g}>\mathfrak{f}_{1}>\ldots>\mathfrak{f}_{r}\right)$ are defined to be the geometric simplexes $\left\|\mathfrak{f}_{1}>\ldots>\mathfrak{f}_{r}\right\|$, and in the case of intersection of such simplexes we have $\varphi_{1} \varphi_{2}=\varphi_{1} \cap \varphi_{2}$. The notion of a butterfly was obtained when we analysed the proof of the theorem in $\S 6.2$ of [B]. In the rough draft version all butterflies are homeomorphic to balls.

In $\S 2$ we prove Theorem $\mathrm{C}$ and some assertions which follow from it.

In $\S 3$ we introduce a convenient model of the sphere $\mathcal{S}$.

In $\S$ 团 we give the basic definitions.

In $\S \S$ 56, after the formula for the intersection of butterflies, we prove theorems on retractions. Every theorem is stated in the same way for the rough and fine versions (the versions differ only in the definitions of the butterflies), and the statement uses an upper semilattice $\mathcal{K}$ of subalgebras of the Lie algebra $\mathfrak{g}$ satisfying fairly general conditions.

In $\S \S 6.26 .3$ we apply the theorems on retractions, in particular, to semilattices $\mathcal{K}$ of types 1)-4). Here we give the final definition of the space $X_{\varepsilon}$ with an estimate for the parameter $\varepsilon$ and deduce that it satisfies the conditions in the statements of Theorem 5.48 and Corollary 5.49 in [B], that is, it is a suitable extension of the space of non-toral directions. We also add a supplement on the compactness of the families of toral and non-toral $H$-subalgebras.

\section{$\S$ 2. Proof of Theorem C. A variant graph theorem}

This section follows on from the Introduction and is not used in the rest of the paper. Here we prove Theorem Cland its corollaries; we also look at the examples of homogeneous Einstein spaces mentioned above.

We keep the notation from $\S 1$. In particular, $Q \in \mathcal{M}_{1}^{G}$ is the normal Riemannian metric on $G / H$ associated with an $\operatorname{Ad}(G)$-invariant Euclidean metric on $\mathfrak{g}, \quad \Sigma$ is the unit sphere in the tangent space to $\mathcal{M}_{1}^{G}$, and $\mathcal{S} \simeq \Sigma$ is the space of geodesic rays on $\mathcal{M}_{1}^{G}$ going out of $Q$. We point out that $\operatorname{sc}(Q)>0$, the scalar curvature of the metric $Q$ is positive if $G / H$ is different from a torus. Throughout, $n=\operatorname{dim}(G / H)$.

2.1. Proof of the criterion. In $[\mathrm{B}$ the main theorem was essentially derived from properties (a)-(d) stated in the Introduction. Theorem $\mathrm{C}$ can be obtained in exactly the same way.

By Theorems $\mathrm{A}$ and $\mathrm{B}$ the compact semi-algebraic set $X_{\varepsilon}$ that will be constructed later has properties (a)-(c) (see Theorem 6.1 and also formula (6.1)).

Recall inequality (d).

Lemma 2.1. Let $\mathcal{K}$ denote the family 1), defined in the Introduction, of all non-toral $H$-subalgebras of the Lie algebra $\mathfrak{g}$, and $\|\mathcal{K}\|$ the corresponding compact set which can be naturally embedded into the space $\mathcal{S}$ of geodesic rays going out of $Q$. Then 
(d) for every number $t \geq 0$ and every ray $r \in\|\mathcal{K}\|$, the inequality $\operatorname{sc}(r(t))>c e^{t / n}>$ 0 holds, where $c$ is a constant independent of $r$ and $t$, and $t \mapsto r(t)$ is the natural parametrization.

The lemma follows easily from another estimate for the scalar curvature at the end of the proof of Proposition 5.6 in B]. See Remark 2.5 below.

To prove Theorem [C] it is now sufficient to consider the simply connected case and pass from finite $\mathcal{K}$ to compact $\mathcal{K}$ in Böhm's final argument.

Theorem 2.2. Let $G / H$ be a compact connected simply connected isotropically reducible homogeneous Riemannian space, $\mathcal{K}$ a family of subalgebras of the Lie algebra $\mathfrak{g}$ of type $\left.1), 1^{*}\right)$, or 2$)$, and $\|\mathcal{K}\|$ the corresponding compact set. If $\|\mathcal{K}\|$ is non-contractible, there exists an infinite sequence $g_{i} \in \mathcal{M}_{1}^{G}, i=1,2, \ldots$, of invariant Riemannian metrics of volume 1 on $G / H$ with scalar curvatures $\operatorname{sc}\left(g_{i}\right)$ bounded above and below by positive constants satisfying the (Palais-Smale) condition $\left|\operatorname{ric}^{0}\left(g_{i}\right)\right|_{g_{i}} \rightarrow 0$. Then $G / H$ admits an invariant positive-definite Einstein metric.

The special cases $X_{\varepsilon}=\varnothing$ and $X_{\varepsilon}=\mathcal{S}$ do not require a separate proof, but we shall discuss them again in the next subsection. The history of Theorem 2.2 naturally started with these cases (in fact, they were considered by Wang and Ziller in 1986). Both of them are related directly or indirectly to the phenomenon of isotropic irreducibility.

Proof. The following argument is due principally to Böhm 6 It is sufficient to prove the first assertion of the theorem. Then, by the main theorem in [BWZ, the sequence $g_{i}$ has a limit point in $\mathcal{M}_{1}^{G}$ - an Einstein metric.

First we recall some basic facts. The manifold of metrics $\mathcal{M}_{1}^{G}$ is itself a non-compact Riemannian symmetric space. It has finite positive dimension, since $G / H$ is isotropically reducible. The subset of Einstein metrics $\mathcal{E}(G, H) \subset \mathcal{M}_{1}^{G}$ coincides with the set of critical points $K=\{g: \nabla \mathrm{sc}(g)=0\}$ of the function $\operatorname{sc}(g)$ (the Hilbert-Jensen theorem [J]). At every point $g \in \mathcal{N}_{1}^{G}$, the gradient $\nabla \mathrm{sc}$ is naturally identified with the traceless part of the Ricci tensor of the metric $g$ taken with opposite sign:

$$
(\nabla \mathrm{sc})_{g}=\frac{\mathrm{sc}(g)}{n} g-\operatorname{ric}(g) \quad \forall g \in \mathcal{M}_{1}^{G} .
$$

On the left-hand side of this equation there is a tangent vector to $\mathcal{M}_{1}^{G}$, and on the righthand side the tensor field on the Riemannian manifold $(G / H, g)$. The Riemannian metric $(\cdot, \cdot)$ on $\mathcal{M}_{1}^{G}$ is defined in such a way that for every $g$ the square of the norm of the vector on the left-hand side with respect to $(\cdot, \cdot)_{g}$ coincides with the square of the norm of the field on $(G / H, g)$ that is on the right-hand side (that is, of the field - $\operatorname{ric}^{0}(g)$ ) calculated at the point $e H$ and at any other point $x \in G / H$, and then also with the square of the $L^{2}$-norm of this field.

We now derive the theorem from properties (a)-(d). By (b) the subspace $X_{\varepsilon}$ is a retract of a neighbourhood $U \subset \mathcal{S}$. In the special cases $X_{\varepsilon}=\varnothing$ or $\mathcal{S}$ this means that $U=X_{\varepsilon}$. We define three subsets of $\mathcal{M}_{1}^{G}$ by the equations

$$
\begin{aligned}
C & =\left\{r(t): r \in U, t>t_{0}\right\}, & & t_{0}>0, \\
B & =\left\{r\left(T_{0}\right): r \in\|\mathcal{K}\|\right\}, & & T_{0}>t_{0}, \\
M & =\left\{g \in \mathcal{M}_{1}^{G}: \operatorname{sc}(g)>k\right\}, & & k \gg 0 .
\end{aligned}
$$

\footnotetext{
${ }^{6}$ We will give a detailed account of the first part of the proof of Theorem 8.1 in [B]. It can also be used to prove Theorem 1.4 from [B] if the restriction $\mathfrak{n}(\mathfrak{h})=\mathfrak{h}$ on the normalizer of the subalgebra $\mathfrak{h}$ is replaced by the more general condition that $\mathcal{K}$ is finite. (We will get rid of both these restrictions.) In this proof, $X_{\text {ent }}^{\Sigma}$ (the extended space of non-toral directions) denotes the space analogous to $X_{\varepsilon}$, and $X_{G / H}^{\Sigma}$ (the nerve) the image of $\|\mathcal{K}\|$ in $\Sigma$ in the case of family 2 ).
} 
By (a) and (d), there exist $k$ and $T_{0}$ such that $C \supset M \supset B$. We fix such numbers $t_{0}$, $T_{0}$, and $k$. Clearly, $B$ is homeomorphic to $\|\mathcal{K}\|$. By the hypothesis of the theorem, $B$ is a non-contractible compact set. Then by (c), $B$ is not contractible over the cylinder $C$, and therefore not over $M$ either. Now we consider the cone $A$ over $B$ with apex $Q$ :

$$
A=\left\{r(t): r \in\|\mathcal{K}\|, 0 \leq t \leq T_{0}\right\} .
$$

Using (d), this time for small values of $t$, we find that $\operatorname{sc}(g)>0$ for all $g \in A$. In the special case when $X_{\varepsilon}=\varnothing$, we set $A:=\{Q\}$ and observe that $\operatorname{sc}(Q)>0$. Suppose that some continuous map $\Phi: \mathcal{M}_{1}^{G} \rightarrow \mathcal{M}_{1}^{G}$ is a non-decreasing function of the scalar curvature, and the restriction $\left.\Phi\right|_{M}$ is homotopic to $\mathrm{id}_{M}$. Then

$$
\Phi(B) \subset \Phi(M) \subset M, \quad \Phi(A) \not \subset M .
$$

(Otherwise, for $\Phi(A) \subset M$, the subset $B$ would be contractible over $M$ to the point $\Phi(Q)$, which is impossible.) Böhm defines the semigroup of such maps $\Phi_{u}, u \geq 0$, where $\Phi_{0}=$ id. It is generated by a complete vector field $\xi$ on $\mathcal{M}_{1}^{G}$.

Lemma $2.3([\mathrm{~B}])$. On $\mathcal{M}_{1}^{G}$ there exists a smooth (complete) vector field $\xi$ with fixedpoint set $K=\{g: \nabla \mathrm{sc}(g)=0\}$ that increases the scalar curvature $\operatorname{sc}(g)$, is proportional to its gradient, and satisfies the inequality $\left\|\xi_{g}\right\|_{g} \leq 1$ on the whole space of metrics, and the equation $\left\|\xi_{g}\right\|_{g}=1$ on the complement of some ball.

(In the case $K=\varnothing$, which must be excluded, the lemma is obviously satisfied by $\xi=\frac{1}{\|\nabla \mathrm{sc}\|} \nabla \mathrm{sc}$; this is sufficient to prove the theorem.) There exists an infinite sequence of metrics $P_{j} \in A, j=1,2, \ldots$, such that $\Phi_{j}\left(P_{j}\right) \notin M$, that is, $\operatorname{sc}\left(\Phi_{j}\left(P_{j}\right)\right) \leq k$. As $A$ is compact and the scalar curvature is strictly positive on the whole $A$, this sequence has a limit point $g_{0} \in A$ satisfying the inequality $k_{0}:=\operatorname{sc}\left(g_{0}\right)>0$. We construct an integral curve $g_{u}=\Phi_{u}\left(g_{0}\right), u \geq 0$, through $g_{0}$. Then, obviously,

$$
k \geq \operatorname{sc}\left(g_{u}\right)=\lim _{m \rightarrow \infty} \operatorname{sc}\left(\Phi_{u}\left(P_{j_{m}}\right)\right) \geq k_{0}>0 \quad \forall u \geq 0
$$

(that is, our curve does not enter $M$ ) and the integral $\int_{0}^{\infty}(\xi \cdot \mathrm{sc})\left(g_{u}\right) d u \leq k-k_{0}$ converges, where

$$
(\xi \cdot \mathrm{sc})\left(g_{u}\right)=\frac{d}{d u} \mathrm{sc}\left(g_{u}\right)=\left\|\xi_{g_{u}}\right\|_{g_{u}}\left\|(\nabla \mathrm{sc})_{g_{u}}\right\|_{g_{u}} .
$$

Finally, our integral curve contains a sequence of metrics $g_{u(i)}, i=1,2, \ldots, u(i)>i$, satisfying the theorem, and the closure of this curve contains an Einstein metric.

Remark 2.4. Lemma 2.3 follows from the compactness of $K$, which was proved in [BWZ].

Remark 2.5. Using the proof of Proposition 5.6 in [B] (where the notation $\gamma_{v}(t)$ is used for $r(t)$ ), we can prove that a positive constant $c$ exists such that

$$
\operatorname{sc}\left(\gamma_{v}(t)\right) \geq c e^{t / \sqrt{n(n-1)}}>0 \quad \forall t \geq 0, \forall r \in\|\mathcal{K}\| \quad(n=\operatorname{dim} G / H)
$$

To do this, we rewrite the nice inequality for the scalar curvature obtained there in the following form:

$$
\operatorname{sc}\left(\gamma_{v}(t)\right) \geq \sum_{i=1}^{p+1}\left(\mathrm{~s}\left(\mathfrak{k}_{i}\right)-\mathrm{s}\left(\mathfrak{k}_{i-1}\right)\right) e^{-t \widehat{v}_{i}}, \quad \mathfrak{h}=\mathfrak{k}_{0}<\mathfrak{k}_{1}<\ldots<\mathfrak{k}_{p+1}=\mathfrak{g},
$$

where $\widehat{v}_{i}$ and $\mathrm{s}\left(\mathfrak{k}_{i}\right)$ are increasing number sequences

$$
\begin{gathered}
\widehat{v}_{1}<\ldots<\widehat{v}_{p+1}, \quad 0=\mathrm{s}(\mathfrak{h})<\mathrm{s}\left(\mathfrak{k}_{1}\right)<\ldots<\mathrm{s}(\mathfrak{g})=\operatorname{sc}(Q), \\
\widehat{v}_{1}<\frac{-1}{\sqrt{n(n-1)}}, \quad \frac{1}{\sqrt{n(n-1)}}<\widehat{v}_{p+1},
\end{gathered}
$$

and $\operatorname{sc}(Q)=\operatorname{sc}(G / H, Q)>0$ is the scalar curvature of the normal homogeneous metric on $G / H$. The curvature $\mathrm{s}(\mathfrak{g})$ has an explicit expression, due to Wang and Ziller, in terms 
of the Killing forms $B_{\mathfrak{h}}$ and $B_{\mathfrak{g}}$ and the quadratic Casimir element $C=C_{\mathfrak{h}, Q} \in Z(U(\mathfrak{h}))$; see WZ1, Proposition 1.9, or [Be], formula (7.89b) cf. [WZ2, Lemma 1.5 or [B], Lemma 4.16. The other terms in the sequence $\mathrm{s}\left(\mathfrak{k}_{i}\right)$ can be written in the form $\mathrm{s}\left(\mathfrak{k}_{i}\right)=\operatorname{sc}\left(K_{i} / H, Q\right)$ and defined by similar formulae. In general, for any subalgebra $\mathfrak{k}$ of type 1$)$ the scalar curvature $\mathrm{s}(\mathfrak{k})>0$ of a suitable homogeneous space $K / H$ can be defined:

$$
\mathrm{s}(\mathfrak{k})=\operatorname{sc}(K / H, Q)=\frac{1}{4}\left(\operatorname{tr}_{Q}\left(B_{\mathfrak{h}}\right)-\operatorname{tr}_{Q}\left(B_{\mathfrak{k}}\right)+\operatorname{tr}\left(C \mid \mathfrak{m}_{\mathfrak{k}}\right)\right)
$$

(on the other hand, the main point is that this is the scalar curvature of an $n$-dimensional asymptotic homogeneous Riemannian geometry). Here, $\mathfrak{m}_{\mathfrak{k}} \subset \mathfrak{k}$ is the $Q$-orthogonal complement of the subalgebra $\mathfrak{h}, \mathfrak{k}=\mathfrak{h}+\mathfrak{m}_{\mathfrak{k}}$. The function $\mathrm{s}(\mathfrak{k})$ takes finitely many values $\varsigma_{1}<\varsigma_{2}<\ldots$, and satisfies the condition $\mathrm{s}\left(\mathfrak{k}_{1}\right)<\mathrm{s}\left(\mathfrak{k}_{2}\right)$ for $\mathfrak{k}_{1}<\mathfrak{k}_{2}$ on a manifold $\mathcal{K}$ of subalgebras of type $1^{*}$ ) or 2 ). The constant $c$ can be defined as the smallest value $\varsigma_{1}$ of the function $\mathrm{s}(\mathfrak{k})$.

2.2. The special cases $X_{\varepsilon}=\varnothing$ and $X_{\varepsilon}=\mathcal{S}$. We now discuss the extremal cases in Theorem 2.2. We begin with the simple case $X_{\varepsilon}=\mathcal{S}$. In view of the fact that $G / H$ is simply connected, this condition is equivalent to each of the following: $\|\mathcal{K}\|=\mathcal{S}$; every $H$-invariant subspace of $\mathfrak{g} / \mathfrak{h}$ corresponds to a subalgebra in the family $\mathcal{K}$ (so there are no toral $H$-subalgebras); up to finite groups, $G / H$ decomposes into a direct product of simply connected isotropically irreducible homogeneous spaces (clearly, the isotropy representation has simple spectrum); every $H$-invariant subspace of $\mathfrak{g} / \mathfrak{h}$ corresponds to an $H$-invariant subalgebra of the Lie algebra $\mathfrak{g}$; the scalar curvature function $\operatorname{sc}(g), g \in \mathcal{M}_{1}^{G}$, is bounded below by a positive constant (by property (d)). If one of these conditions holds, then the function $\operatorname{sc}(g)$ attains its global minimum on $\mathcal{M}_{1}^{G}$, which is an (obviously, unique) invariant Einstein metric. See WZ2, Theorem 2.1.

Now suppose that $X_{\varepsilon}=\varnothing$. This condition is equivalent to each of the following: $\|\mathcal{K}\|=\varnothing ; \mathcal{K}=\varnothing$; every $H$-invariant subalgebra strictly contained between $\mathfrak{g}$ and $\mathfrak{h}$ is toral (that is, decomposes into a direct sum of the subalgebra $\mathfrak{h}$ and an Abelian subalgebra); the scalar curvature function $\operatorname{sc}(g), g \in \mathcal{M}_{1}^{G}$, is bounded above (by property (a) - the latter equivalence was obtained in [B] ). In [WZ2, Theorems 2.2 and 2.4, Wang and Ziller proved that in the case of connected $G$ and $H$ the function $\operatorname{sc}(g)$ attains its global maximum on $\mathcal{M}_{1}^{G}$, and it is an Einstein metric; Böhm proved this in the general case, in [B], Theorems 1.2 and 5.22 .

2.3. Application of the criterion. We will now prove the version of the Graph Theorem stated after Theorem $\mathrm{C}$ To do this, we consider the family $\mathcal{L}$ of all almost semisimple subalgebras of type $1^{*}$ ) and the subfamily $\mathcal{L}^{\min } \subset \mathcal{L}$ of subalgebras of type 2 ). Let [r] denote the orbit of every subalgebra $\mathfrak{l} \in \mathcal{L}$ with respect to $\operatorname{Norm}_{G}(H)^{0}$. Let $\mathrm{B}_{\mathrm{WZ}}$ denote the graph with vertices $[\mathfrak{l}], \mathfrak{l} \in \mathcal{L}$, and edges $([\mathfrak{k}],[\mathfrak{l}])$, where $\mathfrak{k}<\mathfrak{l}$. Let $\mathrm{B}_{\mathrm{WZ}}^{\min }$ be the subgraph of this graph induced on the subset of vertices $[\mathfrak{k}], \mathfrak{k} \in \mathcal{L}^{\min }$. Then

$$
\mathrm{B}_{\mathrm{WZ}}^{\min } \subset \mathrm{B}_{\mathrm{WZ}} \subset \Upsilon_{\mathrm{WZ}}
$$

Here, $\Upsilon_{\mathrm{WZ}}$ is the part of the Wang-Ziller graph whose disconnectedness, by BWZ, implies that an invariant Einstein metric exists on $G / H$.

Using the property that $\mathcal{L} / \operatorname{Norm}_{G}(H)^{0}$ and $\mathcal{L}^{\mathrm{min}} / \operatorname{Norm}_{G}(H)^{0}$ are finite, we obtain the following.

Lemma 2.6. There exist continuous surjective maps of the compact sets $\|\mathcal{K}\|=\|\mathcal{L}\|$ and $\left\|\mathcal{L}^{\mathrm{min}}\right\|$ onto the flag complexes $K_{\Gamma}$ of the graphs $\Gamma=\mathrm{B}_{\mathrm{WZ}}$ and $\mathrm{B}_{\mathrm{WZ}}^{\min }$, respectively.

Note that $\|\mathcal{L}\|$ and $\left\|\mathcal{L}^{\min }\right\|$ are homotopy equivalent by Theorem $\mathrm{B}$.

Proof. The vertices $\left[\mathfrak{k}_{i}\right], i=1, \ldots, r$, of every complete subgraph $\Sigma \subset \Gamma$ can be ordered in such a way that, without loss of generality, $\operatorname{dim}\left(\mathfrak{k}_{1}\right)>\ldots>\operatorname{dim}\left(\mathfrak{k}_{r}\right)$. Using the finiteness 
property we find by induction that there exist subalgebras $\mathfrak{f}_{i} \in\left[\mathfrak{k}_{i}\right]$ that form a flag $\varphi=\left(\mathfrak{f}_{1}>\ldots>\mathfrak{f}_{r}\right)$. We define a map $q_{\varphi}$ of the simplex $\|\varphi\|$ onto the simplex $|\Sigma|$ by the formula $q_{\varphi}\left(\sum_{i=1}^{r} \lambda_{i} \chi^{\mathfrak{f}_{i}}\right)=\sum_{i=1}^{r} \lambda_{i}\left[\mathfrak{f}_{i}\right]$, where the $\lambda_{i}$ are barycentric coordinates. The map of sets $q=\bigcup_{\varphi \in \Delta(\mathcal{K})} q_{\varphi}:\|\mathcal{K}\| \rightarrow\left|K_{\Gamma}\right|$ is well defined. We consider the inverse image $Z=q^{-1}(|\Sigma|)$ of every simplex $|\Sigma|$ of the complex $\left|K_{\Gamma}\right|$. We denote the flag manifold by $Y \subset\left[\mathfrak{k}_{1}\right] \times \ldots \times\left[\mathfrak{k}_{r}\right]$ and observe that the natural projection of $Y \times|\Sigma|$ onto $|\Sigma|$ can be factored through a continuous map of $Y \times|\Sigma|$ onto $Z$ and the restriction $\left.q\right|_{Z}$. Consequently, every $Z$ is compact and the restriction of $q$ to $Z$ is continuous, while the number of such subsets $Z$ is finite. Hence $q$ is continuous.

This lemma and Theorem C imply the following.

Corollary 2.7. If either of the graphs $\mathrm{B}_{\mathrm{WZ}}$ or $\mathrm{B}_{\mathrm{WZ}}^{\min }$ is disconnected, then there exists an invariant Einstein metric on $G / H$.

As was observed in [BWZ, Proposition 4.9, if $G / H$ has a finite fundamental group and $\operatorname{dim}(\mathfrak{z}(\mathfrak{g})) \geq 1$, then the graph $\Upsilon_{\text {WZ }}$ contains at most one connected component. Then the criterion based on the disconnectedness of the graph $\Upsilon_{\mathrm{WZ}}$ gives nothing, at least for $\Upsilon_{\mathrm{WZ}} \neq \varnothing$, but the criterion based on the disconnectedness of its subgraphs is effective.

On the other hand, as noted in [B], Corollary 7.6, if all the subalgebras $\mathfrak{k}<\mathfrak{g}$ of type 4 ) are contained in just one proper subalgebra, then the corresponding simplicial scheme $\Delta(\mathcal{K})$ is contractible.

Using these two criteria, one can construct homogeneous spaces $G / H$ with disconnected graph $\mathrm{B}_{\mathrm{WZ}}^{\mathrm{min}}$ for which, based on the original criteria in $\mathrm{BWZ}$ and $\mathrm{B}$, nothing can be said, while the preceding corollary asserts the existence of invariant Einstein metrics.

Example 2.8 (13 July 2011). Let $G=\mathrm{U}_{N}$ be the group of unitary transformations of the space $V=\mathbb{C}^{N}=\bigvee^{p} \mathbb{C}^{3}$, for $N=\left(\begin{array}{c}p+2 \\ 2\end{array}\right)$; let $\mathrm{U}_{N-1}$ be the stabilizer of the vector $v=\otimes^{p} e_{3} \in V$; let $R: \mathrm{U}_{3} \rightarrow G$ be the $p$ th symmetric power of the basic representation of the group $\mathrm{U}_{3}, p>1$; let $K=R\left(\mathrm{U}_{3}\right)$ and let $H=K \cap \mathrm{U}_{N-1}$ be a four-dimensional subgroup, so that $G / H$ is a compact homogeneous space of dimension $>31$. We observe that $\mathfrak{h}<\mathfrak{k}<\mathfrak{g}$ and $\mathfrak{k}=\mathfrak{u}_{3}=\mathfrak{h}+\mathfrak{s u}_{3}$ is both a maximal subalgebra and a minimal non-toral $H$-subalgebra. Hence $[\mathfrak{k}]$ is an isolated vertex of the graph $\mathrm{B}_{\mathrm{WZ}}^{\min }$ (it is easy to see that it is not unique); this graph is disconnected, and by the preceding corollary there exists an invariant Einstein metric on $G / H$. We now attempt to use the criteria in BWZ and [B]. Obviously, $V$ splits into pairwise non-equivalent irreducible $H$-modules $V_{k}, \operatorname{dim}_{\mathbb{C}} V_{k}=k+1, k=0, \ldots, p$. Hence the centralizer of $H$ in $G$ is a torus $T^{p+1}$, and $[\mathfrak{k}]$ is its $p$-dimensional orbit $\operatorname{Ad}\left(T^{p+1}\right) \mathfrak{k}$. Hence the family of subalgebras 2$)$ is infinite, and it makes sense to state Böhm's criterion for family 4). By decomposing $\mathfrak{g}$ with respect to representations of the torus $T^{p+1}$, it is easy to prove that every subalgebra of type 4 ) is contained in $\mathfrak{u}_{N-1}$. Then Böhm's polyhedron is contractible by the criterion stated above ( $\mathrm{B}]$, Corollary 7.6), and, what is more, for $p=2$ it consists of the single point $\left[\mathfrak{h}+\mathfrak{s u}\left(V_{1}\right)\right]$. Next, $\operatorname{dim}(\mathfrak{z}(\mathfrak{g}))=1$, and so the Wang-Ziller graph is connected by Proposition 4.9, [BWZ]. Therefore, neither of the criteria in [BWZ] and [B] works in the case under consideration 7

Now let $Z \subset G$ be a closed connected commutative normal subgroup, let $\bar{G}=G / Z$ and $\bar{H}=H / H \cap Z$. It is easy to verify that the compact sets $\|\mathcal{L}\|$ and $\left\|\mathcal{L}^{\text {min }}\right\|$ are

${ }^{7}$ When I constructed Example 2.8 I started from Example 6.3 in BWZ. See also $\S 3$ in BK]. 
preserved under the passage from $G, H$ to $\bar{G}, \bar{H}$ if the fundamental group $\pi_{1}(G / H)$ is finite.

Theorem C implies the following.

Corollary 2.9. If either $\|\mathcal{L}\|$ or $\left\|\mathcal{L}^{\min }\right\|$ is non-contractible, an invariant Einstein metric exists on both $G / H$ and $\bar{G} / \bar{H}$.

As an important example we consider the quotient space

$$
\bar{G} / \bar{H}=\left(G_{1} / H_{1} \times \ldots \times G_{p} / H_{p}\right) / Z,
$$

where $p \geq 2$ and every $G_{i} / H_{i}$ is a simply connected homogeneous Einstein space. For $i=1$ we impose the condition $\widetilde{H}_{*}\left(\left\|\mathcal{L}_{1}^{\min }\right\|\right) \neq 0$, where $\widetilde{H}_{q}$ means the $q$ th singular homology group reduced modulo a point. Suppose that for every $i>1$ the group $G_{i} / H_{i}$ is the principle circle bundle over an irreducible Hermitian symmetric space equipped with the natural Sasaki-Einstein geometry with full isometry group $G_{i}$. We have $\left\|\mathcal{L}^{\text {min }}\right\|=$ $\left\|\mathcal{L}_{1}^{\min }\right\| * S^{p-2}$ (see the next remark). Hence, $\widetilde{H}_{q}\left(\left\|\mathcal{L}^{\min }\right\|\right)=\widetilde{H}_{q-p+1}\left(\left\|\mathcal{L}_{1}^{\min }\right\|\right)$ for every $q$ (see, for example, Theorem 2 in $\S 13$ of A. T. Fomenko and D. B. Fuks, A course in homotopic topology. Textbook, Nauka, Moscow, 1989 (Russian)). Hence $\bar{G} / \bar{H}$ is a homogeneous Einstein space.

It is possible to construct similar examples by replacing Hermitian symmetric spaces by homogeneous Kähler-Einstein spaces. There are homeomorphisms

$$
\|\mathcal{L}\|=\left\|\mathcal{L}_{1}\right\| * \ldots *\left\|\mathcal{L}_{p}\right\| * S^{p-2},
$$

and the same for $\left\|\mathcal{L}^{\min }\right\|$. Then $\widetilde{H}_{*}(\|\mathcal{L}\|)=\widetilde{H}_{*}\left(\left\|\mathcal{L}^{\text {min }}\right\|\right)$ can be expressed in terms of reduced homology groups of the factors, using the well-known generalization of Künneth's formula to joins.

Remark 2.10. Let $G / H=G_{1} / H_{1} \times \ldots \times G_{p} / H_{p}$ be the Cartesian product of $p \geq 2$ nontrivial homogeneous spaces $\left(G=G_{1} \times \ldots \times G_{p}, H=H_{1} \times \ldots \times H_{p}, H_{i} \varsubsetneqq G_{i}\right)$. Then under the condition $\left|\pi_{1}(G / H)\right|<\infty$, we have

$$
\|\mathcal{L}\| \supset\left\|\mathcal{L}_{1}\right\| * \ldots *\left\|\mathcal{L}_{p}\right\| * S^{p-2}
$$

(where $S^{k}$ is the $k$-dimensional sphere and $*$ is the join of topological spaces). Furthermore, if $\mathfrak{g}_{i}+\mathfrak{h} \in \mathcal{L}^{\text {min }}, i=1, \ldots, p$, then

$$
\left\|\mathcal{L}^{\min }\right\| \supset\left\|\mathcal{L}_{1}^{\min }\right\| * \ldots *\left\|\mathcal{L}_{p}^{\min }\right\| * S^{p-2} .
$$

The inclusions (2.1) and (2.2) can be replaced by homeomorphisms if every almost semisimple subalgebra $\mathfrak{k} \in \mathcal{L}$ is equal to the sum of its projections onto the $\mathfrak{g}_{i}$. For example, $\|\mathcal{L}\|=\left\|\mathcal{L}^{\min }\right\|=S^{p-2}$ for $G / H=\left(\mathrm{SU}_{2} / T^{1}\right)^{p}$.

Remark 2.11. These properties are similar to the following theorem in [B], which concerns families $\mathcal{K}, \mathcal{K}_{i}$ of subalgebras of type 4 ) (recall that such families are finite):

$$
\|\mathcal{K}\| \approx\left\|\mathcal{K}_{1}\right\| * \ldots *\left\|\mathcal{K}_{p}\right\| * S^{p-2} .
$$

Here $\approx$ can be correctly understood as a homeomorphism if all the $\left\|\mathcal{K}_{i}\right\|$ are noncontractible, and in the general case as a homotopy equivalence 8 We point out that when $\mathfrak{g}_{i_{0}}+\mathfrak{h} \notin \mathcal{K}$, the polyhedra $\left\|\mathcal{K}_{i_{0}}\right\|$ and $\|\mathcal{K}\|$ are contractible. For example, when $\# \mathcal{K}_{1}=\# \mathcal{K}_{2}=1, p=2$, the polyhedra $\|\mathcal{K}\|$ and $\left\|\mathcal{K}_{1}\right\| *\left\|\mathcal{K}_{2}\right\|$ are homeomorphic to segments, and $\left\|\mathcal{K}_{1}\right\| *\left\|\mathcal{K}_{2}\right\| * S^{0}$ to a suspension over a segment, that is, to a square.

\footnotetext{
${ }^{8}$ In the original statement, 2.3 is just a homeomorphism. In the proof for $p=2$ there all the cases which are omitted were mentioned, namely, the join $J=\left\|\mathcal{K}_{1}\right\| *\left\|\mathcal{K}_{2}\right\|$ of two simplicial complexes and cones over $J$.
} 


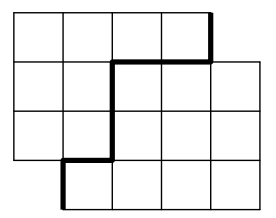

The scheme explains the triangulation of the suspension $J * S^{0}$ of the join $J$ of two ordered simplexes of dimensions 3 and 2. The boldface broken line, staircase, defines one of the maximal simplexes of the triangulation.

Figure 1. Towards the proof of (2.3),$p=2$.

This case is possible. We have $\# \mathcal{K}=\# \mathcal{L}=1$ for each of the following 11-dimensional homogeneous Einstein spaces discovered by Wang:

$$
\begin{aligned}
& \mathrm{SU}(3) \times \mathrm{SU}(3) / \Delta \mathrm{SU}(2)(\mathrm{U}(1) \times \mathrm{U}(1)), \\
& \mathrm{Sp}(2) \times \mathrm{Sp}(2) / \Delta \mathrm{SU}(2)(\mathrm{Sp}(1) \times \mathrm{Sp}(1)), \\
& \mathrm{SU}(3) \times \mathrm{Sp}(2) / \Delta \mathrm{SU}(2)(\mathrm{U}(1) \times \mathrm{Sp}(1)) .
\end{aligned}
$$

An example of an effective use of theorem (2.3) is given on the last page in [BK]. The proof of this theorem can be illustrated by Figure 1.

\section{$\S$ 3. The Convention about the Choice of Model}

We now return to the main sequence of results of this paper. We let $G$ denote a compact Lie group, and $H$ a compact subgroup of it that does not contain connected normal subgroups. For a given $\operatorname{Ad}(G)$-invariant Euclidean metric $Q$ on the Lie algebra $\mathfrak{g}$, we will also use $Q$ to denote the associated Riemannian metric on $G / H$. We assume that $Q \in \mathcal{M}_{1}^{G}$.

The set $\mathcal{S}$ of geodesic rays on $\mathcal{M}_{1}^{G}$ going out of $Q$ is considered as a topological sphere, and we define two models of this sphere:

1st model: the unit sphere $\Sigma$ of the Euclidean space of $\operatorname{Ad}(H)$-invariant symmetric operators with trace 0 on $\mathfrak{g} / \mathfrak{h}$;

2nd model: the boundary $\mathrm{S}=\mathrm{S}[\mathfrak{h}]$ of the homogeneous ball $\mathrm{D}^{*}[\mathfrak{h}]$ consisting of all positive-definite $\operatorname{Ad}(H)$-invariant symmetric operators with trace 1 on $\mathfrak{g} / \mathfrak{h}$.

The first model is used in [BWZ] and [B], while we use the second to simplify notation. This leads to different descriptions of the same subspaces of the sphere $\mathcal{S}$ ( $W^{\Sigma}$ and others) and other superficial differences. We should explain that the first, original, model for $\mathcal{S}$ is constructed naturally, while the second is connected to it by a homeomorphism $\mathrm{h}: \mathrm{S} \rightarrow \Sigma$. Here are the formulae for $\mathrm{h}$ and $\mathrm{h}^{-1}$ :

$$
v=\mathrm{h}(A)=\frac{A-1_{\mathfrak{g} / \mathfrak{h}} / n}{\sqrt{|A|^{2}-1 / n}}, \quad A=\mathrm{h}^{-1}(v)=\frac{1}{n}\left(1_{\mathfrak{g} / \mathfrak{h}}-\frac{v}{\lambda(v)}\right),
$$

where $\lambda(v)$ is the smallest eigenvalue of the traceless operator $v, \lambda(v)<0$, and $n=$ $\operatorname{dim}(G / H)$.

This immediately shows, for example, that every Böhm stratum $W^{\Sigma}(\mathfrak{k}) \subset \Sigma$ and its closure $X^{\Sigma}(\mathfrak{k})$ are contained in an open half-sphere, which fact was not noticed in $[\mathrm{B}$. The definition of $X^{\Sigma}(\mathfrak{k})$ is given in the next section.

In what follows we will not normally mention the difference between models 1 and 2 .

\section{$\S$ 4. Definitions}

The space of filtering linear operators on the Lie algebra g. In Böhm's paper on homogeneous Einstein metrics [B], it was proved that there exist strong deformation retractions of the form $C \rightarrow B$, where $C$ is a compact subspace of the topological sphere $\mathrm{S}$ and $B \subset C$ is a simplicial complex. This complex can be constructed starting from a 
certain 9 set of subalgebras $\mathfrak{f} \supset \mathfrak{h}$ of the Lie algebra $\mathfrak{g}$. The vertices of the complex $B$ are the linear operators $\bar{\chi}^{\mathfrak{f}}:=\frac{1}{\operatorname{dim}(\mathfrak{g} / \mathfrak{f})}\left(1_{\mathfrak{g}}-1_{\mathfrak{f}}\right)$ (see below). The simplexes of the complex $B$ are the rectilinear simplexes

$$
\mid \varphi /=\text { Convex hull }\left\{\bar{\chi}^{f_{i}}, i=1, \ldots, r\right\},
$$

where $\varphi=\left(\mathfrak{f}_{1}>\ldots>\mathfrak{f}_{r}\right)$ is a decreasing flag of subalgebras $\mathfrak{f}_{i}, \mathfrak{g}>\mathfrak{f}_{i}>\mathfrak{h}$. The whole space $C \supset B$ (up to the homeomorphism h: $\mathrm{S} \rightarrow \Sigma$ ) is contained in the intersection of the sphere $\mathrm{S}$ and the compact set $\mathfrak{F}_{+}$, which we will now proceed to define. To do this we need the notion of a filtering linear operator on $\mathfrak{g}$.

We fix a Euclidean scalar product $Q: \mathfrak{g} \times \mathfrak{g} \rightarrow \mathbb{R}$ on the compact Lie algebra $\mathfrak{g}$ satisfying the identity $Q([X, Y], Z) \equiv Q(X,[Y, Z])$. A linear operator $A: \mathfrak{g} \rightarrow \mathfrak{g}$ is called symmetric if $Q(A X, Y) \equiv Q(X, A Y)$. We associate the family of vector subspaces $F_{a}$ indexed by any numbers $a \in \mathbb{R}$

$$
F_{a}=\operatorname{span}\{X \in \mathfrak{g}: \exists r \leq a, A X=r X\}
$$

with $A$. Then $F_{a} \subset F_{b}$ for $a \leq b$. We say that a symmetric operator $A$ is filtering if $\left(F_{a}\right)_{a \in \mathbb{R}}$ is a filtration of the Lie algebra $\mathfrak{g}$, that is, the following equivalent conditions hold:

1) $\left[F_{a}, F_{b}\right] \subset F_{a+b}$ for any $a, b \in \mathbb{R}$,

2) for every $X, Y \in \mathfrak{g}$ the $\operatorname{limit}_{\lim _{t \rightarrow+\infty}} e^{t A}\left[e^{-t A} X, e^{-t A} Y\right]$ exists,

3) $\left|C_{i, j}^{k}\right|^{2}\left(\lambda_{k}-\lambda_{i}-\lambda_{j}\right) \leq 0$ for the eigenvalues $\lambda_{i}$ of the operator $A$ and structure constants of the Lie algebra $\mathfrak{g}$ with respect to an eigenbasis.

For example, with every subalgebra $\mathfrak{k} \subsetneq \mathfrak{g}$ we can associate the filtering operator $\bar{\chi}^{\mathfrak{k}}$ defined by the formula

$$
\bar{\chi}^{\mathfrak{k}}:=\frac{1}{\operatorname{dim}(\mathfrak{g} / \mathfrak{k})}\left(1_{\mathfrak{g}}-1_{\mathfrak{k}}\right) .
$$

It corresponds to the following filtration $\left(F_{a}\right)_{a \in \mathbb{R}}$ :

$$
F_{a}=\left\{\begin{array}{l}
0 \text { for } a<0 \\
\mathfrak{k} \text { for } 0 \leq a<\frac{1}{\operatorname{dim}(\mathfrak{g} / \mathfrak{k})}, \\
\mathfrak{g} \text { for } \frac{1}{\operatorname{dim}(\mathfrak{g} / \mathfrak{k})} \leq a
\end{array}\right.
$$

We note that for every filtering operator $A$,

$$
F_{0} \supset \operatorname{ker}(A) \supset\left[F_{0}, F_{0}\right], \quad\left[A, \operatorname{ad}\left(F_{0}\right)\right]=0 .
$$

Let $\mathfrak{F}_{+}$denote the topological space of all filtering non-negative symmetric operators $A$ with trace 1 on $\mathfrak{g}$. Then $\bar{\chi}^{\mathfrak{k}} \in \mathfrak{F}_{+}$.

Main Lemma 4.1. The space $\mathfrak{F}_{+}$is a compact semi-algebraic set.

Proof. We write down a system of polynomial inequalities for $\mathfrak{F}_{+}$. With every operator $A \in \mathfrak{F}_{+}$we associate the chain $v_{1}=-A . c, \ldots, v_{i+1}=-A . v_{i}, i=1,2, \ldots$, of vectors in the space $\mathfrak{g} \otimes \wedge^{2} \mathfrak{g}^{*}$ :

$$
c=[\cdot, \cdot], \quad v_{1}=-A . c=[A \cdot, \cdot]+[\cdot, A \cdot]-A[\cdot, \cdot], \ldots .
$$

Then $-A$ induces a symmetric operator with simple strictly positive spectrum on $V=$ $\operatorname{span}\left\{v_{i}, i=1,2, \ldots\right\}$. We form the sequence $D_{k}, k=1,2, \ldots$, of principle corner minors of the infinite matrix $\left(a_{i, j}\right)$ of scalar products $a_{i, j}=a_{i+j-1,1}=\left(-A . v_{i}, v_{j}\right)$, $i, j \in\{1,2, \ldots\}$. We have $0<a_{i, j} \leq 2^{i+j+1}|c|^{2}$, and so by the formula for the volumes of parallelepipeds, $0 \leq D_{i} \leq a_{i, i} D_{i-1} \leq 2^{2 i+1}|c|^{2} D_{i-1} \leq E_{0}^{-i} D_{i-1}$ (where $E_{0}$ is a number

\footnotetext{
${ }^{9}$ The subalgebras $\mathfrak{f}$ used in Böhm's final constructions correspond to closed subgroups of the compact Lie group $G$ with Lie algebra $\mathfrak{g}$ and satisfy a number of other conditions.
} 
independent of $A$ ). Conversely, every symmetric operator $A$ on $\mathfrak{g}$ satisfying a system of non-strict polynomial inequalities with fixed $E>0$

$$
D_{i}(A) \geq E^{i+1} D_{i+1}(A) \geq 0, \quad i=1,2, \ldots,
$$

satisfies the strict inequalities $D_{i}(A)>0, i=1, \ldots, \operatorname{dim}(V)$, and so by Sylvester's theorem, $(-A . v, v)>0$ for all $v \in V \backslash 0$; such an operator $A$ is filtering, which proves the assertion.

Note that if $G / H$ is compact and simply connected, the set $\mathfrak{F}_{+}$is completely determined by the preceding inequalities and the equations $A \mathfrak{h}=0, \operatorname{trace}(A)=1, E=E_{0}$ (that is, the condition that the spectrum of $A$ be non-negative follows from these).

The compact space $\mathrm{W} \simeq W^{\Sigma}$ of degenerate filtrations. A filtering operator $A \in \mathfrak{F}_{+}$ is said to be degenerate with respect to a proper subalgebra $\mathfrak{h}<\mathfrak{g}$ if

$$
\mathfrak{h}<F_{0}=\operatorname{ker}(A)<\mathfrak{g} .
$$

We fix a compact group $\mathcal{A}$ of automorphisms of the algebra $\mathfrak{g}$ preserving $\mathfrak{h}$ and $Q$ (the $\operatorname{group} \operatorname{Ad}(H)$ and its toral extension, for example, occur in $[\mathrm{B}]$ ).

Let $\mathrm{W}$ denote the space of all $\mathcal{A}$-invariant filtering operators $A \in \mathfrak{F}_{+}$that are degenerate with respect to the subalgebra $\mathfrak{h}$.

Obviously, W inherits the properties of being compact and semi-algebraic from $\mathfrak{F}_{+}$.

Lemma 4.2. The kernel $\mathfrak{k} \subset \mathfrak{g}$ of every operator $A \in \mathrm{W}$ is an $\mathcal{A}$-invariant subalgebra contained strictly between $\mathfrak{g}$ and $\mathfrak{h}$, that is, $\mathfrak{h}<\mathfrak{k}<\mathfrak{g}$.

In [B] the compact topological space $W^{\Sigma}$, which is (semi-algebraically) homeomorphic to the space $\mathrm{W}$, is studied in detail. (The space $W^{\Sigma}$ is contained in the sphere $\{A: A \mathfrak{h}=0$, $\left.\operatorname{trace}(A)=0, \operatorname{trace}\left(A^{2}\right)=1\right\}$. We use the non-spherical model $\mathrm{W}$ of this space; the spherical model is obtained from it by simple arithmetical operations.)

Following $[B]$, we define the partition of the compact set $W$ into subsets $X^{*}[\mathfrak{k}]$ of operators with fixed kernel $F_{0}=\mathfrak{k}$. The closure of each of these subsets is expressed by the formula $\mathrm{X}[\mathfrak{k}]=\{A \in \mathrm{W}: A \mathfrak{k}=0\}$. It is identified with the compact starshaped semi-algebraic set $X^{\Sigma}(\mathfrak{k}) \subset W^{\Sigma}$ defined in $[\mathrm{B}, \S 5.4$.

Rough draft version of the space $W^{\Sigma}$. We define the rough draft version of the space $W^{\Sigma}$ to be the (compact) topological space of all $(\mathcal{A}, \mathfrak{h})$-invariant non-negative symmetric operators $A$ with trace 1 on $\mathfrak{g}$ satisfying the conditions $A X=0, A \operatorname{ad}(X)=\operatorname{ad}(X) A$ for all $X \in \mathfrak{h}$ and some $X \in \mathfrak{g}, X \notin \mathfrak{h}$.

The rough draft version $W_{\text {draft }}$ of the topological space $W^{\Sigma}$ is a union of closed cells (homeomorphic to balls) such that a non-empty intersection of cells is a cell, and every cell $D$ properly contained in a cell $D_{1}$ is contained in the boundary sphere of the ball $D_{1}$ (generally speaking, the boundary sphere is not covered by such cells $D$ ). Cells correspond uniquely to $(\mathcal{A}, \mathfrak{h})$-invariant subalgebras $\mathfrak{k}, \mathfrak{h}<\mathfrak{k}<\mathfrak{g}$, and are denoted by $\mathrm{D}[\mathfrak{k}]$; they can be defined by the equations

$$
\mathrm{D}[\mathfrak{k}]=\left\{A \in \mathrm{W}_{\text {draft }}: A \mathfrak{k}=0,[A, \operatorname{ad}(\mathfrak{k})]=0\right\} .
$$

The space $W_{\text {draft }}$ is contained in the boundary sphere of the new ball $\mathrm{D}[\mathfrak{h}]$, that is, strictly speaking, of the convex compact set defined as the set of all symmetric linear operators with trace 1 and non-negative eigenvalues that annihilate the $\mathcal{A}$-invariant subalgebra $\mathfrak{h}<\mathfrak{g}$ and commute with $\mathcal{A}$ and $\operatorname{ad}(\mathfrak{h})$. In the formula for $\mathrm{D}[\mathfrak{k}]$ one can replace $W_{\text {draft }}$ by $D[\mathfrak{h}], \mathfrak{h} \geq 0$.

The space $\mathrm{W} \simeq W^{\Sigma}$ is embedded into its rough draft version in such a way that the intersection of $\mathrm{W}$ with every cell $\mathrm{D}[\mathfrak{k}]$ is expressed by the formula

$$
\mathrm{D}[\mathfrak{k}] \cap \mathrm{W}=\mathrm{X}[\mathfrak{k}]:=\{A \in \mathrm{W}: A \mathfrak{k}=0\} .
$$


The subset $\mathrm{X}[\mathfrak{k}]$ is starshaped, compact, and semi-algebraic. The centre of the star $\mathrm{X}[\mathfrak{k}] \simeq$ $X^{\Sigma}(\mathfrak{k})$ can be regarded as the centre of the ball $\mathrm{D}[\mathfrak{k}]$ and is situated at the point

$$
A=\bar{\chi}^{\mathfrak{k}} \text {. }
$$

The star $\mathrm{X}[\mathfrak{k}]$ contains the standard Euclidean ball

$$
\left\{A \in \mathrm{D}[\mathfrak{k}]:\left|A-\bar{\chi}^{\mathfrak{k}}\right| \leq \frac{1}{3 \operatorname{dim}(\mathfrak{g} / \mathfrak{k})}\right\} \text {. }
$$

These properties of $\mathrm{X}[\mathfrak{k}]$ will be explained in the next subsection.

A quite different version of the space $W^{\Sigma}$ is considered in the following remark.

Remark 4.3 (See BWZ, $\S 3$ ). We construct the BWZ-hull $\mathrm{E}$ of the subset $\mathrm{W}$ in the boundary sphere $\mathrm{S}$ of the ball $\mathrm{D}[\mathfrak{h}]$. We fix a sequence of real numbers $b_{0}=0<a_{1}<$ $b_{1}<\ldots<a_{k-1}<b_{k-1}=1 / n=\frac{1}{\operatorname{dim}(G / H)}$ such that every time $2 a_{i}<b_{i}$. We say that an interval $\left[a_{i}, b_{i}\right], i=1, \ldots, k-1$, is empty if it does not contain eigenvalues of the operator $A$ and therefore, $F_{a_{i}}=F_{2 a_{i}}$. For a sufficiently large $k$, for every $A \in \mathrm{S}$ there exists at least one empty interval $\left[a_{i}, b_{i}\right]$. We set $A \in \mathrm{E}$ if, for every empty interval $\left[a_{i}, b_{i}\right]$, the subspace $F_{a_{i}} \subset \mathfrak{g}$ is a subalgebra, that is, $\left[F_{a_{i}}, F_{a_{i}}\right] \subset F_{2 a_{i}}$. Hence, $\mathrm{W} \subset \mathrm{E} \subset \mathrm{S}$. For $\mathcal{A}=\operatorname{Ad}(H)$ and $a_{i}-b_{i-1}=\delta / n>0$, the topological space $\mathrm{E}$ is homeomorphic to the one denoted by $\bigcup W_{i}^{\Sigma}$ in [BWZ] (where $\alpha_{i}=1-n b_{k-1-i}$ ).

The construction of filtering operators and the starshapedness of $X[\mathfrak{k}]$. We need to explain why $\mathrm{X}[\mathfrak{k}]:=\mathrm{D}[\mathfrak{k}] \cap \mathfrak{F}_{+}$is starshaped. First of all we introduce the Euclidean norm $|A|=\sqrt{\operatorname{trace}\left(A^{2}\right)}$ and observe that for $\mathfrak{k} \geq \mathfrak{h} \geq 0$ and $\mathrm{D}[\mathfrak{k}] \neq\left\{\chi^{\mathfrak{k}}\right\}$ the subset

$$
\Omega=\left\{A \in \mathrm{D}[\mathfrak{k}]:\left|A-\bar{\chi}^{\mathfrak{k}}\right|=\frac{1}{k \sqrt{5-1 / k}}\right\},
$$

where $k=\operatorname{dim}(\mathfrak{g} / \mathfrak{k})$, of the compact $\mathrm{D}[\mathfrak{k}]$ is a Euclidean sphere.

For filtering operators $A \in \mathfrak{F}_{+}$the following construction is possible.

Lemma 4.4. The subset $\Omega$ satisfies $\Omega \subset \mathfrak{F}_{+}$, and for every $A \in \Omega$ there exists a number $t_{A} \geq 1$ such that

$$
A_{t}=t\left(A-\bar{\chi}^{\mathfrak{k}}\right)+\bar{\chi}^{\mathfrak{k}} \begin{cases}\in \mathfrak{F}_{+} & \text {if } 0 \leq t \leq t_{A}, \\ \notin \mathfrak{F}_{+} & \text {if } t_{A}<t<\infty .\end{cases}
$$

Hence $\mathrm{X}[\mathfrak{k}]$ is starshaped and contains the Euclidean ball bounded by the sphere $\Omega$.

Proof. Let $A \in \Omega$, and let $\vec{\lambda}=\left(\lambda_{1}, \ldots, \lambda_{k}\right)$ be the tuple of eigenvalues of the restriction $\left.A\right|_{\mathfrak{k} \perp}$. We have $k>1$, since $\Omega \neq \varnothing$. We now verify that $\lambda_{i}-2 \lambda_{j} \leq 0$ for $i \neq j$. Then the operator $A$ will be filtering in view of the conditions $A \mathfrak{k}=0$ and $[A, \operatorname{ad}(\mathfrak{k})]=0$. For definiteness we set $i=1, j=k$ and consider the $k$-dimensional vector

$$
\vec{\mu}=k\left(1+\frac{1}{k}, \frac{1}{k}, \ldots, \frac{1}{k}, \frac{1}{k}-2\right) .
$$

We have $|\vec{\mu}|=1 / R$, where $R$ is the radius of the sphere $\Omega$. Hence, $k\left(\lambda_{1}-2 \lambda_{k}\right)+1=$ $(\vec{\lambda} \mid \vec{\mu}) \leq 1=R|\vec{\mu}|$. Using the vectors $\vec{\mu}$ obtained from (4.5) by any permutations of coordinates, we obtain that $\lambda_{i}-2 \lambda_{j} \leq 0$ for all $i \neq j$. Hence, $A \in \mathfrak{F}_{+}$. It remains to use the following obvious property:

(*) if a straight line $L$ connects different commuting operators $A^{\prime}, A^{\prime \prime} \in \mathfrak{F}_{+}, A^{\prime} A^{\prime \prime}=$ $A^{\prime \prime} A^{\prime}$, then $L \cap \mathfrak{F}_{+}$is a segment.

Therefore $\left\{t \geq 0: \bar{\chi}^{\mathfrak{k}}+t\left(A-\bar{\chi}^{\mathfrak{k}}\right) \in \mathfrak{F}_{+}\right\}$is a segment containing 0 and 1 . This proves (4.4). 
Where semi-algebraicity is used. The properties of being semi-algebraic and compact can be verified for various subsets of the space of symmetric linear operators which occur in what follows. For example, for the similar balls $\mathrm{D}[\mathfrak{h}]$ and $\mathrm{D}[\mathfrak{k}]$ these properties follow immediately from the definitions. The union $W_{\text {draft }}=\bigcup_{\mathfrak{h}<\mathfrak{k}} \mathrm{D}[\mathfrak{k}]=$ $\operatorname{pr}_{1}\{(A, X) \in \mathrm{D}[\mathfrak{h}] \times(\mathfrak{g} / \mathfrak{h}):|X|=1, A X=0, A \operatorname{ad}(X)=\operatorname{ad}(X) A\}$ is also a compact semialgebraic set. This follows from the Tarski-Seidenberg theorem, which states that the projection of $\mathbb{R}^{p} \times \mathbb{R}^{q}$ onto the first factor takes semi-algebraic subsets to semi-algebraic ones. That $\mathrm{W}$ and $\mathrm{X}[\mathfrak{k}]$ are semi-algebraic follows immediately from the semi-algebraicity of $\mathfrak{F}_{+}$. And so on.

Apart from these verifications, semi-algebraic geometry is essential to the proofs of the following two simple lemmas.

Let $d(u, v)$ denote the Euclidean distance on $\mathbb{R}^{N}$.

Lemma 4.5 ( $\delta$-neighbourhoods). Let $X$ and $T \subset X$ be non-empty compact semialgebraic subsets of $\mathbb{R}^{N}$. Suppose that for every $\delta>0$ there exists a continuous semialgebraic map $f: X \times[0,1] \rightarrow X,(x, t) \mapsto f_{t}(x)$, satisfying the conditions

$$
\begin{aligned}
& f_{t}(x)=x \text { for } x \in T, t \in[0,1] \text { and for } x \in X, t=0 ; \\
& d\left(f_{t}(x), T\right) \leq \delta \text { for } x \in X, t=1 .
\end{aligned}
$$

Then $T$ is a semi-algebraic strong deformation retract of $X$.

Proof. By the well-known general theorem of semi-algebraic geometry, there exists a semi-algebraic homeomorphism of a finite simplicial complex $|K|$ onto $X$ such that $T$ is a union of simplexes ([BC], Theorem 9.2.1). Hence $T$ can be regarded as a strong deformation retract of a compact regular neighbourhood $U$ of itself in the second barycentric subdivision of the complex $|K|$. We denote the semi-algebraic strong deformation retraction of $U$ to $T$ by $G(x, t), x \in U, t \in[0,1]$. For sufficiently small $\delta$ the neighbourhood $U$ contains $U_{\delta}=\{x \in X: d(x, T) \leq \delta\}$. (Cf. also the proof of [BC], Proposition 9.4.4.) Hence there exists a function $F: X \times[0,1] \rightarrow X$ of the form

$$
F(x, t)= \begin{cases}f_{2 t}(x) & \text { if } 0 \leq t \leq 1 / 2 \\ G\left(f_{1}(x), 2 t-1\right) & \text { if } 1 / 2 \leq t \leq 1 .\end{cases}
$$

The function $F(x, t)$ is continuous and semi-algebraic, since both properties also hold for its restrictions to the closed subsets $\{t \leq 1 / 2\}$ and $\{t \geq 1 / 2\}$ of $X \times[0,1]$. Consequently, $F$ defines a semi-algebraic strong deformation retraction of $X$ onto $T$. The lemma is proved.

Let $X=\mathrm{D}[\mathfrak{k}]$ or $\mathrm{X}[\mathfrak{k}]$, let $S$ be the boundary sphere of the ball $D=\mathrm{D}[\mathfrak{k}]$, let $Y \subset S \cap X$ be a closed semi-algebraic subset (say, $Y=S \cap \mathrm{X}[\mathfrak{k}]=\bigcup_{\mathfrak{l}>\mathfrak{k}} \mathrm{X}[\mathfrak{l}]$ ), and $T$ the union of segments connecting the centre of the ball with points $y \in Y$. In the special cases where $Y=\varnothing$ or $D$ reduces to a point, we define $T$ to be the centre of the ball $D$.

Lemma 4.6 (On retraction). Under these conditions the topological space $T$ is a strong deformation retract of the space $X$ (the deformation is semi-algebraic).

See a similar assertion in $[\mathrm{B}$, Theorem 5.39.

The lemma remains true for any compact starshaped semi-algebraic subset $X$ of the ball $D$. We will prove the lemma. Using the convexity of $D$, we represent every point $x \in D$ in the form $r \omega, r \in[0,1], \omega \in S$, where for convenience the centre of the ball $D$ and of the star $X$ is placed at the point 0 . On the boundary sphere $S$ there exists a continuous semi-algebraic function $s(\omega)=\min \left(1, \delta^{-1} d(\omega, Y)\right.$ ) (see Proposition 2.2.8 
in $[\mathrm{BC}])$. For every $t \in[0,1]$, let $f_{t}(r \omega)=(1-s(\omega) t) r \omega$. Then $f_{t}(X) \subset X$ as $X$ is starshaped. Obviously, for $t=1$ for every $x=r \omega \in X$ there exists $\alpha \in Y$ such that

$$
d\left(f_{1}(r \omega), T\right) \leq d\left(f_{1}(r \omega),(1-s(\omega)) r \alpha\right)=(1-s(\omega)) s(\omega) \delta r \leq \frac{1}{4} \delta .
$$

It remains to use Lemma 4.5 above.

\section{$\S 5$. Retraction theorems. Butterflies}

The admissible polyhedron $/ \mathcal{K} /$. With every decreasing flag $\varphi=\left(\mathfrak{f}_{0}, \ldots, \mathfrak{f}_{r}\right)$ of subalgebras of the compact Lie algebra $\mathfrak{g}$ such that $\mathfrak{g}>\mathfrak{f}_{i}>\mathfrak{f}_{i+1}$, we can associate the Euclidean convex hull

$$
|\varphi|:=\text { Convex hull }\left\{\bar{\chi}^{f_{i}}: i=0, \ldots, r\right\}
$$

of the set of linear symmetric operators $\bar{\chi}^{f_{i}}, i=0, \ldots, r$. This hull is an $r$-dimensional simplex, since the differences $\bar{\chi}^{f_{i}}-\bar{\chi}^{f_{i+1}}$ are pairwise orthogonal.

With every finite set $\mathcal{K}$ whose elements are $(\mathcal{A}, \mathfrak{h})$-invariant subalgebras $\mathfrak{k}$ such that $\mathfrak{h}<\mathfrak{k}<\mathfrak{g}$, we can now associate a compact (non-convex) polyhedron $/ \mathcal{K} /$ contained in $\mathrm{W} \simeq W^{\Sigma}$. To do this we order $\mathcal{K}$ by inclusion of subalgebras and denote the set of well-ordered subsets $\varphi$ in $\mathcal{K}$, that is, flags of subalgebras in $\mathcal{K}$, by $\Delta(\mathcal{K})$ (as usual). We claim that

$$
|\varphi / \cap / \psi|=\mid \varphi \cap \psi / \quad \forall \varphi, \psi \in \Delta(\mathcal{K})
$$

see Proposition 6.4 in $\mathrm{B}$ or formula (5.2) below. (To verify this we can represent every symmetric operator with non-negative spectrum $\left(\lambda_{1} \geq \ldots \geq \lambda_{n}\right)$, where $\lambda_{n}=0$, in the form of the sum $A=\sum_{i=1}^{n-1}\left(\lambda_{i}-\lambda_{i+1}\right)\left(1_{\mathfrak{g}}-1_{F_{\lambda_{i+1}}}\right)$. Obviously, this representation is unique.) The subsets $\varphi \in \Delta(\mathcal{K})$ can be regarded as simplexes of an abstract simplicial complex, which is again denoted by $\Delta(\mathcal{K})$. Then, by (5.1),

the rectilinear simplexes $\mid \varphi /, \varphi \in \Delta(\mathcal{K})$, form a geometric realization of the order complex $\Delta(\mathcal{K})$, that is, the compact polyhedron $/ \mathcal{K} /:=\bigcup_{\varphi \in \Delta(\mathcal{K})} / \varphi /$.

It is easy to verify that the polyhedron $/ \mathcal{K} /$ is contained in the non-spherical model $\mathrm{W}$ of the space $W^{\Sigma}$, that is, all its points are filtering non-negative symmetric $(\mathcal{A}, \mathfrak{h})$-invariant linear operators with trace 1 on the Lie algebra $\mathfrak{g}$ that are degenerate with respect to the subalgebra $\mathfrak{h}$.

The polyhedron $/ \mathcal{K} /$ is said to be admissible if the finite set $\mathcal{K} \cup(\mathfrak{g})$ is an upper semilattice of subalgebras, that is, together with any subalgebras $\mathfrak{k}$ and $\mathfrak{l}$ it also contains the smallest subalgebra containing them, $\mathfrak{f}=\sup (\mathfrak{k}, \mathfrak{l})$.

The first retraction theorem. Retraction to an admissible polyhedron. Let $\mathcal{K} \ni \mathfrak{g}$ be a finite upper semilattice of $(\mathcal{A}, \mathfrak{h})$-invariant subalgebras $\mathfrak{k}$ such that $\mathfrak{h}<\mathfrak{k} \leq \mathfrak{g}$, and let $\mathcal{K}^{\#}:=\mathcal{K} \backslash(\mathfrak{g})$.

Theorem 5.1. The admissible polyhedron $/ \mathcal{K}^{\#} /$ is a strong deformation retract of the compact topological space $\mathrm{X}[\mathcal{K}]:=\bigcup_{\mathfrak{k} \in \mathcal{K} \#} \mathrm{X}[\mathfrak{k}]$.

This theorem is a natural generalization of Theorem $6.10 \mathrm{in}[\mathrm{B}]$. The following theorem is the rough draft version.

Theorem 5.2. The admissible polyhedron $/ \mathcal{K}^{\#} /$ is a strong deformation retract of the compact topological space $\mathrm{D}[\mathcal{K}]:=\bigcup_{\mathfrak{k} \in \mathcal{K} \#} \mathrm{D}[\mathfrak{k}]$. 
Both theorems are valid in the semi-algebraic category. In what follows we give the scheme of the proof, which is just a new version of the proof of Theorem 6.10 in $[\mathrm{B}$, (though it appears to be noticeably different).

In the proof Böhm used a covering of the space $\mathrm{X}[\mathcal{K}]$ by compact subsets that are intermediate between simplexes $/ \varphi /, \varphi \in \Delta\left(\mathcal{K}^{\#}\right)$, and members $\mathrm{X}[\mathfrak{k}]$ of the original covering. He outlined a useful algebro-topological construction, finding the intersections of some of them. We combine these objects and simplexes under the general name butterflies and place them at the foundation of our exposition. The definitive algebraic formula for the intersection of butterflies, which was not given in Böhm's paper, is presented below.

Description of butterflies. Butterflies are compact semi-algebraic subsets of the topological space $\mathrm{W} \simeq W^{\Sigma}$ or its rough draft version $\mathrm{W}_{\text {draft }}$, respectively. They are divided into three types.

First type. A butterfly of this type is defined by the equation $B[\mathfrak{f}]=X[\mathfrak{f}]$ orB $[\mathfrak{f}]=D[\mathfrak{f}]$, respectively, for every $(\mathcal{A}, \mathfrak{h})$-invariant subalgebra $\mathfrak{f}, \mathfrak{h}<\mathfrak{f}<\mathfrak{g}$; furthermore, $\mathrm{B}[\mathfrak{g}]:=\varnothing$.

In general, butterflies are associated with flags $\varphi=\left(\mathfrak{f}_{1}, \ldots, \mathfrak{f}_{r}\right)$ of $(\mathcal{A}, \mathfrak{h})$-invariant subalgebras of the algebra $\mathfrak{g}$, where $\mathfrak{g} \geq \mathfrak{f}_{i}>\mathfrak{f}_{i+1}>\mathfrak{h}, r \geq 1$. The case $r=1$ has already been discussed.

Second type. For $r>1$ and $\mathfrak{g} \in \varphi$ a butterfly is defined as the $(r-2)$-dimensional rectilinear simplex $/ \varphi \backslash \mathfrak{g} /=$ Convex hull $\left\{\widehat{\chi}^{f_{i}}: i=2, \ldots, r\right\}$.

Third type. For $r>1$ and $\mathfrak{g} \notin \varphi$ a butterfly is defined as the union of all segments of the Euclidean space with the left endpoint at a point of the butterfly $\mathrm{B}\left[\mathfrak{f}_{1}\right]$ and the right endpoint at a point of the $(r-2)$-dimensional simplex

$$
/ \varphi \backslash \mathfrak{f}_{1} /=\text { Convex hull }\left\{\bar{\chi}^{f_{i}}: i=2, \ldots, r\right\} .
$$

We claim that these segments can only intersect at endpoints, that is, the following lemma holds.

Lemma 5.3. A butterfly of the third type is the join $X * Y$ of two butterflies of the first and second types $X=\mathrm{B}\left[\mathfrak{f}_{1}\right]$ and $Y=/ \varphi \backslash \mathfrak{f}_{1} /$.

The butterfly of a flag $\varphi$ is denoted by $\mathrm{B}[\varphi]=\mathrm{X}[\varphi]$ or $\mathrm{D}[\varphi]$, respectively, depending on the version. By Lemma [5.3, in the rough draft version every butterfly $\mathrm{D}[\varphi]$ is homeomorphic to an $(r+k-1)$-dimensional ball, where $k=\operatorname{dim} \mathrm{D}\left[\mathfrak{f}_{1}\right] \geq-1$.

Algebraic formula for intersection of butterflies. As we can show,

$$
\mathrm{B}\left[\varphi_{1}\right] \cap \mathrm{B}\left[\varphi_{2}\right]=\mathrm{B}\left[\varphi_{1} \varphi_{2}\right],
$$

the intersection of butterflies $\mathrm{B}\left[\varphi_{1}\right]$ and $\mathrm{B}\left[\varphi_{2}\right]$ is again a butterfly $\mathrm{B}[\varphi]$ of some flag $\varphi=$ $\varphi_{1} \varphi_{2}$, for which a formula will be written. Furthermore, $\varphi$ is the supremum of the flags $\varphi_{i}, i=1,2$, with respect to the non-standard partial order relation on the set of flags: $\psi>\varphi$ if there exists a sequence of flags $\psi=\varphi_{1}, \ldots, \varphi_{k}=\varphi$ such that the largest subalgebras $\max \left(\varphi_{i}\right)$ form a non-increasing sequence, $\max \left(\varphi_{i}\right) \geq \max \left(\varphi_{i+1}\right)$, the lengths of adjacent flags differ by $\pm 1,\left|\ell\left(\varphi_{i}\right)-\ell\left(\varphi_{i+1}\right)\right|=1$, and for every $i<k$ either $\varphi_{i+1} \subset \varphi_{i}$, $\max \left(\varphi_{i}\right)>\max \left(\varphi_{i+1}\right)$, or $\varphi_{i+1} \supset \varphi_{i}, \max \left(\varphi_{i}\right)=\max \left(\varphi_{i+1}\right)$. (We write $\psi \subset \varphi$ if every subalgebra in the sequence $\psi$ occurs in the sequence $\varphi$.)

In short, $\psi \geq \varphi$ if and only if the following conditions hold:

$$
\max (\psi) \geq \max (\varphi) ; \quad \mathfrak{l} \in \psi \text { and } \mathfrak{l} \notin \varphi \text { imply } \mathfrak{l}>\max (\varphi)
$$

Proposition 5.4. The intersection of any butterflies $\mathrm{B}[\varphi]$ and $\mathrm{B}[\psi]$ is again a butterfly. Namely, $\mathrm{B}[\varphi] \cap \mathrm{B}[\psi]=\mathrm{B}[\varphi \psi]$, where $(\varphi, \psi) \mapsto \varphi \psi$ is the following composition of wellordered subsets of the lattice of all $\mathcal{A}$-invariant subalgebras:

$$
\varphi \psi:=(\psi \cap \varphi) \cup\{\mathfrak{f} \in \varphi: \mathfrak{f}>\max \psi\} \cup\{\mathfrak{f} \in \psi: \mathfrak{f}>\max \varphi\} \cup\{\sup (\varphi \cup \psi)\} ;
$$




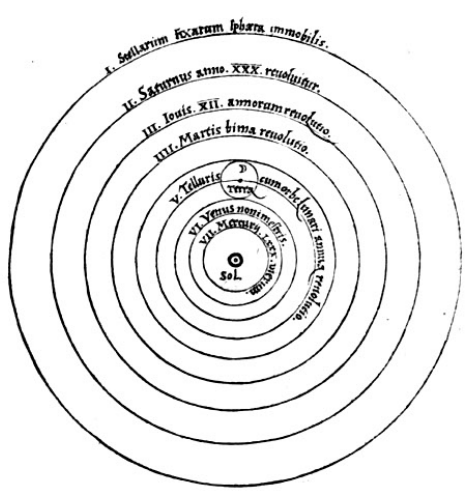

The points $\bar{\chi}^{k}$ belong to a finite set of Euclidean spheres with centre $\bar{\chi}^{h}$ (Sun), and $h(\mathfrak{k})=\operatorname{dim}(\mathfrak{k})$ increases as the radius of the sphere increases. Butterflies of the first type are contained in the corresponding tangent planes, so that the whole Universe is contained in the ball of radius 1 , and the union of butterflies of the first type with $h>s$ is contained in the spherical shell.

Figure 2. "Copernicus' Universe" (to the filtration $X^{(s)}$ ).

where $\cap$ and $\cup$ are the set-theoretic operations of intersection and union.

For example, for two butterflies of the first type, we claim that $B[\mathfrak{f}] \cap B[l]=B[\sup (\mathfrak{f}, \mathfrak{l})]$; see (4.1) and (4.2).

Proof. It easily follows from $\psi \geq \varphi$ that $\mathrm{B}[\psi] \subset \mathrm{B}[\varphi]$, and so $\mathrm{B}\left[\varphi_{1} \varphi_{2}\right] \subset \mathrm{B}\left[\varphi_{i}\right], i=1,2$. Now let $\varphi_{1} \neq \varphi_{2}, \varphi_{i} \neq(\mathfrak{g})$. We prove the inclusion $\mathrm{B}\left[\varphi_{1}\right] \cap \mathrm{B}\left[\varphi_{2}\right] \subset \mathrm{B}\left[\varphi_{1} \varphi_{2}\right]$ by induction on $\lambda=\ell\left(\varphi_{1}\right)+\ell\left(\varphi_{2}\right)$, where $\ell(\varphi)$ is the length of every flag $\varphi$. First we consider the following cases:

1) $\lambda=2$, that is, $\ell\left(\varphi_{1}\right)=\ell\left(\varphi_{2}\right)=1$; then the assertion is easily reduced to the definition of butterflies of the first type;

2) $\ell\left(\varphi_{2}\right)=1, \ell\left(\varphi_{1}\right)>1, \varphi_{2}=(\mathfrak{f}), \min \left(\varphi_{1}\right) \geq \mathfrak{f}$; then $\varphi_{1} \varphi_{2}=\varphi_{1}$, that is, $\varphi_{1} \geq \varphi_{2}$, and the assertion is obvious (similarly in the case where $\varphi_{1}$ and $\varphi_{2}$ are interchanged);

3) $\ell\left(\varphi_{1}\right)>1, \ell\left(\varphi_{2}\right)>1, \mathfrak{f}:=\min \left(\varphi_{1}\right)=\min \left(\varphi_{2}\right)$; then $\varphi_{i}=\left(\psi_{i}, \mathfrak{f}\right), i=1,2$, whence $\varphi_{1} \varphi_{2}=\left(\psi_{1} \psi_{2}, \mathfrak{f}\right)$; the butterflies $\mathrm{B}\left[\psi_{i}\right], i=1,2$, are contained in the boundary sphere $\mathrm{S}[\mathfrak{f}]$ of the ball $\mathrm{D}[\mathfrak{f}]$ (here, $\mathrm{S}[\mathfrak{f}] \neq \varnothing$ as $\psi_{1} \neq \psi_{2}$ ), and the point $\chi^{\mathfrak{f}} \in \mathrm{D}^{*}[\mathfrak{f}]=\mathrm{D}[\mathrm{f}] \backslash \mathrm{S}[\mathrm{f}]$ can be taken as the centre of this ball, cf. (4.3); hence the induction hypotheses implies that

$$
\mathrm{B}\left[\varphi_{1}\right] \cap \mathrm{B}\left[\varphi_{2}\right]=\left(\mathrm{B}\left[\psi_{1}\right] * \bar{\chi}^{f}\right) \cap\left(\mathrm{B}\left[\psi_{2}\right] * \bar{\chi}^{\mathfrak{f}}\right)=\mathrm{B}\left[\psi_{1} \psi_{2}\right] * \bar{\chi}^{\mathrm{f}}=\mathrm{B}\left[\varphi_{1} \varphi_{2}\right] .
$$

(Here $\mathrm{B} * a$ is the cone with apex $a$ and base $\mathrm{B}$, and by definition, $\mathrm{B}[\mathfrak{g}] * \bar{\chi}^{\mathfrak{f}}=\varnothing * \bar{\chi}^{\mathfrak{f}}=\bar{\chi}^{\mathfrak{f}}$.) In the other cases, for every $A \in \mathrm{B}\left[\varphi_{1}\right] \cap \mathrm{B}\left[\varphi_{2}\right]$, up to interchanging $\varphi_{1}$ and $\varphi_{2}$, there exists a subflag $\psi \subset \varphi_{1}, \psi>\varphi_{1}$, such that $A \in \mathrm{B}[\psi]$. Then $\ell(\psi)<\ell\left(\varphi_{1}\right)$ and by the induction hypotheses, $A \in \mathrm{B}\left[\psi \varphi_{2}\right] \subset \mathrm{B}\left[\varphi_{1} \varphi_{2}\right]$.

The rule for the intersection of the butterflies $\mathrm{X}[\varphi]$ and $\mathrm{X}[\psi]$ was obtained by Böhm under certain restrictions on the flags $\varphi$ and $\psi$. See $[\mathrm{B}, \S 5.4$ and $[\mathrm{B}$, Proposition 6.4, for butterflies of the first and second types, and see [B], Lemma 6.9 for butterflies of the third type.

Scheme of the proof of the first theorem. We give an outline of the proofs of Theorems 5.1 and 5.2, which are analogous. We want to prove a sequence of semialgebraic strong deformation retractions exists (where semi-algebraic indicates the semialgebraic category)

$$
\rho^{(s)}: X^{(s-1)} \stackrel{\text { onto }}{\longrightarrow} X^{(s)}, \quad s=1, \ldots, m,
$$

with $X^{(0)}=\mathrm{B}[\mathcal{K}]$ and $X^{(m)}=/ \mathcal{K}^{\#} /$. Let $h(\mathfrak{k})=\operatorname{dim}(\mathfrak{k})=\operatorname{dim}(\mathfrak{g})-\left|\bar{\chi}^{\mathfrak{k}}\right|^{-2}$ for every subalgebra $\mathfrak{k} \in \mathcal{K}$. Instead of this, we can fix any strictly increasing function $h: \mathcal{K} \rightarrow$ $\{1,2, \ldots\}$, that is, such that $\mathfrak{f}<\mathfrak{l}$ implies $h(\mathfrak{f})<h(\mathfrak{l})$. We extend the monotonic function 
$h$ from $\mathcal{K}$ to $\Delta(\mathcal{K})$ by setting $h(\varphi):=h(\max (\varphi))$ for every flag $\varphi \in \Delta(\mathcal{K})$ (this gives $h(\varphi) \leq h(\psi)$ for $\varphi<\psi)$ and set by definition

$$
X^{(s)}=\bigcup_{\varphi \in \Delta(\mathcal{K}): h(\varphi)>s} \mathrm{~B}[\varphi], \quad s \in\{0,1, \ldots\} .
$$

Then $X^{(0)}=\mathrm{B}[\mathcal{K}]$, and for $m=h(\mathfrak{g})-1$,

$$
X^{(m)}=/ \mathcal{K}^{\#} /:=\bigcup_{\varphi \in \Delta(\mathcal{K}): \max (\varphi)=\mathfrak{g}} \mathrm{B}[\varphi] .
$$

We observe that the butterflies $\mathrm{B}[\varphi]$ are compact semi-algebraic sets and so, because $\Delta(\mathcal{K})$ is finite, their unions and intersections are too. It follows from the formula for the intersection of butterflies that

$$
\mathrm{T}[\varphi]:=\mathrm{B}[\varphi] \cap X^{(h(\varphi))}=\bigcup_{\psi \in \Delta(\mathcal{K}): \psi>\varphi, h(\psi)>h(\varphi)} \mathrm{B}[\psi]
$$

holds for every flag $\varphi \in \Delta(\mathcal{K})$.

We claim that the butterfly B $[\varphi]$ of every flag $\varphi$ contracts over itself to its "body" $\mathrm{T}[\varphi]$ (that is, the body is its semi-algebraic strong deformation retract). For a flag $\varphi=(\mathfrak{f})$ of length 1, up to notation this is the statement of Lemma 4.6 above on retraction. The general case can be easily reduced to this special case. As a result, the butterfly $\mathrm{B}[\varphi] \subset X^{(s-1)}$ with $h(\varphi)=s$ is contracted to its intersection with $X^{(s)}$. Developing this argument, it is possible to obtain the retraction of $X^{(s-1)}$ to $X^{(s)}$ we seek.

Instead, we could apply our Lemma 4.5] on $\delta$-neighbourhoods directly to the pair of spaces $X^{(s)} \subset X^{(s-1)}$. A map $f: X^{(s-1)} \times[0,1] \rightarrow X^{(s-1)}$ satisfying the conditions of the lemma can be constructed quite explicitly, not just in the finite case, but even in the case of a compact semilattice $\mathcal{K}$.

The construction of $f: X^{(s-1)} \times[0,1] \rightarrow X^{(s-1)}$ and the completion of the proof. We denote the natural (orthogonal) projection of every butterfly $\mathrm{B}[\varphi]$ onto the underlying simplex $\mathrm{B}[\varphi \cup(\mathfrak{g})] \subset \mathrm{B}[\varphi]$ by $\gamma_{\varphi}$. Namely, let $\varphi=\left(\mathfrak{f}_{1}>\ldots>\mathfrak{f}_{r}\right)$ be a flag of subalgebras of length $r \geq 1$. For $\mathfrak{f}_{1}=\mathfrak{g}$ we set $\gamma_{\varphi}=$ id. For $\mathfrak{f}_{1} \neq \mathfrak{g}$ every element $x \in \mathrm{B}[\varphi]$ admits the representation

$$
x=\lambda_{1} z+\sum_{i=2}^{r} \lambda_{i} \bar{\chi}^{\mathfrak{f}_{i}} ; \quad z \in \mathrm{B}\left[\mathfrak{f}_{1}\right] ; \quad \lambda_{i} \geq 0, \quad \sum_{i=1}^{r} \lambda_{i}=1
$$

and $\gamma_{\varphi}(x)=\sum_{i=1}^{r} \lambda_{i} \bar{\chi}^{f_{i}}$. We now fix a function $\sigma: X^{(s-1)} \rightarrow[0,1]$ satisfying the condition $\sigma(x)=0$ for every $x \in X^{(s)}$. Then we can define a map $f_{t, \varphi}: \mathrm{B}[\varphi] \rightarrow \mathrm{B}[\varphi]$ by

$$
f_{t, \varphi}(x)=(1-\sigma(x) t) x+\sigma(x) t \gamma_{\varphi}(x) \quad \forall x \in \mathrm{B}[\varphi] .
$$

We claim that, even in the case of infinite $\mathcal{K}$, for every $t \in[0,1]$ there exists a (discontinuous) map

$$
f_{t}=\bigcup_{\varphi \in \Delta(\mathcal{K}): h(\varphi) \geq s} f_{t, \varphi}: X^{(s-1)} \rightarrow X^{(s-1)} .
$$

To prove this, we only need to verify that the point $\gamma_{\varphi}(x)$ is independent of the flag $\varphi$ for $\sigma(x) \neq 0$, that is, for $x \in X^{(s-1)} \backslash X^{(s)}$.

For every $x \in X^{(0)}$, let $\psi_{x}$ denote the largest flag $\psi \in \Delta(\mathcal{K})$ satisfying the condition $x \in \mathrm{B}[\psi]$. We use the non-standard order relation on the set of flags defined above. The existence of $\psi_{x}$ follows from the ascending chain condition for the partially ordered set $\Delta(\mathcal{K})$. By the formula for intersections of butterflies, $\mathrm{B}\left[\psi_{x}\right]$ is the smallest butterfly $\mathrm{B}[\varphi]$, $\varphi \in \Delta(\mathcal{K})$, containing $x$. We introduce the notation

$$
\gamma(x)=\gamma_{\psi_{x}}(x), \quad h(x)=h\left(\psi_{x}\right)=\max \left\{k: x \in X^{(k-1)}\right\} .
$$


It follows from the conditions $\psi_{x} \geq \varphi$ and $h\left(\psi_{x}\right)=h(\varphi)=s$ that $\psi_{x} \subset \varphi$ (that is, every subalgebra of the flag $\psi_{x}$ is contained in the flag $\varphi$ ) and $\mathfrak{f}_{1}=\max (\varphi)=\max \left(\psi_{x}\right)$, and this implies $\gamma_{\varphi}(x)=\gamma(x)$. Hence,

$$
h(x)=s \Longrightarrow \gamma_{\varphi}(x)=\gamma(x) \quad \forall \varphi, x \in \mathrm{B}[\varphi], h(\varphi)=s .
$$

Formula (5.4) proves the map $f_{t}: X^{(s-1)} \rightarrow X^{(s-1)}$ exists (not a priori semi-algebraic and discontinuous). Furthermore, $f_{0}: X^{(s-1)} \rightarrow X^{(s-1)}$ and the restriction of $f_{t}$ to $X^{(s)}$, $t \in[0,1]$, are identity maps.

We now turn to the definition of the function $\sigma(x), x \in X^{(s-1)}$. We define it by the equation

$$
\sigma(x):=\min \left(1, \delta^{-1} d(\widetilde{x}, Z)\right) .
$$

The following notation is used in the definition (5.5): $\widetilde{x}=(x, \gamma(x))$ and $Z$ is the set of all pairs $\left(x^{\prime}, y^{\prime}\right)$, where $x^{\prime} \in X^{(s)}, y^{\prime}=\gamma_{\varphi}\left(x^{\prime}\right)$ for some flag $\varphi \in \Delta(\mathcal{K})$ such that $h(\varphi) \in\{s, s+1, \ldots\}, x^{\prime} \in \mathrm{B}[\varphi]$; the distance between pairs $(x, y)$ and $\left(x^{\prime}, y^{\prime}\right)$ is defined as the maximum of the Euclidean distances $d\left(x, x^{\prime}\right)$ and $d\left(y, y^{\prime}\right)$, that is,

$$
d\left((x, y),\left(x^{\prime}, y^{\prime}\right)\right)=\max \left(d\left(x, x^{\prime}\right), d\left(y, y^{\prime}\right)\right),
$$

and the distance from $\widetilde{x}=(x, \gamma(x))$ to the set $Z$ is defined naturally.

For $x \in X^{(s)}$ (that is, for $h(x)>s$ ) we have $\widetilde{x} \in Z$, and then $\sigma(x)=0$.

We can now write down the final formula for $f_{t}$ :

$$
f_{t}(x)=(1-\sigma(x) t) x+\sigma(x) t \gamma(x) \quad \forall x \in X^{(s-1)} \quad(t \in[0,1]) .
$$

We claim that for $t=1$ the function $g=f_{1}$ satisfies the condition

$$
d\left(g(x), X^{(s)}\right)<\delta \quad \forall x \in X^{(s-1)} .
$$

We will use the fact that the Hausdorff distance between segments of Euclidean space satisfies the inequality

$$
d^{H}\left([x, y],\left[x^{\prime}, y^{\prime}\right]\right) \leq \max \left(d\left(x, x^{\prime}\right), d\left(y, y^{\prime}\right)\right) .
$$

(Obviously, this is not always the case in spherical Riemannian geometry; therefore if we were to work with the spherical Böhm model we would need additional arguments!) By definition, $g(x)$ lies on the segment $[x, \gamma(x)]$. On the other hand, if $x^{\prime} \in X^{(s)}$ and $x^{\prime} \in \mathrm{B}[\varphi]$, then the segment $\left[x^{\prime}, \gamma_{\varphi}\left(x^{\prime}\right)\right]$ is also contained in $X^{(s)}$. Consequently, we have

$$
\begin{gathered}
\sigma(x)<1 \Longrightarrow d\left(g(x), X^{(s)}\right) \leq d(\widetilde{x}, Z)<\delta, \\
\sigma(x)=1 \Longrightarrow g(x)=\gamma(x) \in X^{(m)} \subset X^{(s)} .
\end{gathered}
$$

To complete the proof of Theorems 5.1 and 5.2 it remains to verify that the map (5.6) is continuous and semi-algebraic and use Lemma 4.5 on $\delta$-neighbourhoods (for $X=X^{(s-1)}$ and $T=X^{(s)}$ ). If (as is easy to verify) these properties hold for the restriction of (5.6) to every subspace $\mathrm{B}[\varphi] \times[0,1]$ for every flag $\varphi \in \Delta(\mathcal{K})$, then as $\mathcal{K}$ is finite they also hold for (5.6).

Moreover, the following generalization holds.

Proposition 5.5. Suppose that the semilattice $\mathcal{K} \ni \mathfrak{g}$, fixed above, of some subalgebras of the Lie algebra $\mathfrak{g}$ is a compact, but not necessarily finite semi-algebraic subset of the (disconnected) Grassmannian of vector subspaces of the space $\mathfrak{g}$, and the strictly increasing function $h: \mathcal{K} \rightarrow\{1,2, \ldots\}$ is continuous, for example, $h(\mathfrak{k})=\operatorname{dim}(\mathfrak{k})$. Define the spaces $X^{(s)}$ in terms of $\mathcal{K}$ and $h$ as above. Then

1) all the $X^{(s)}, s=0, \ldots, m$, are also compact semi-algebraic sets;

2) equation (5.6) defines a continuous semi-algebraic map $f: X^{(s-1)} \times[0,1] \rightarrow X^{(s-1)}$ for every $s \in\{1, \ldots, m\}$; 
3) every $X^{(s)}, s \in\{1, \ldots, m\}$, is a semi-algebraic strong deformation retract of the space $X^{(s-1)}$.

Proof. 1) By hypothesis, $\Delta(\mathcal{K})$ is contained in the disconnected flag manifold $F=$ $\bigcup_{r>0} \bigcup_{\operatorname{dim}(\mathfrak{g}) \geq k_{1}>\ldots>k_{r}} F_{k_{1}, \ldots, k_{r}}(\mathfrak{g})$ and is a compact semi-algebraic subset. We now consider the sets of pairs

$$
Y=\{(x, \varphi): \varphi \in \Delta(\mathcal{K}), x \in \mathrm{B}[\varphi]\}\}, \quad Y^{(s)}=\{(x, \varphi) \in Y: h(\varphi) \geq s+1\},
$$

where $s=1, \ldots, m$. Then $X^{(s)}=p Y^{(s)}$, where $p(x, \varphi)=x$. By manipulating the natural vector bundles over $F_{k_{1}, \ldots, k_{r}}(\mathfrak{g})$, it is easy to prove that $Y=\bigcup Y_{k_{1}, \ldots, k_{r}}$ is compact and semi-algebraic. (In the X-version, we also need to use our main Lemma 4.1] on the compactness and semi-algebraicity of the set $\mathfrak{F}_{+}$of filtering non-negative symmetric operators with trace 1 on $\mathfrak{g}$.) Next, $Y^{(s)}$ is the union of connected components of the space $Y$. Consequently, $Y^{(s)}$, and then, by the Tarski-Seidenberg theorem, also $X^{(s)}=p Y^{(s)}$ are again compact and semi-algebraic.

2) We now verify the second assertion. First of all consider $\gamma_{\varphi}: \mathrm{B}[\varphi] \rightarrow \mathrm{B}[\varphi \cup(\mathfrak{g})]$. Recall that by definition this is an orthogonal projection, and $\gamma_{\varphi}(x)$ is the nearest point of the simplex $\mathrm{B}[\varphi \cup(\mathfrak{g})]$ to $x \in \mathrm{B}[\varphi]$. Consequently, $(x, \varphi) \in Y^{(s-1)} \mapsto \gamma_{\varphi}(x)$ is a continuous semi-algebraic map. Then $Z=\left\{\left(x, \gamma_{\varphi}(x)\right):(x, \varphi) \in Y^{(s-1)}, x \in X^{(s)}\right\}$ is a semi-algebraic set (since $Y^{(s-1)}$ and $X^{(s)}$ are) and

$$
\varsigma(x, \varphi):=\min \left(1, \delta^{-1} d\left(\left(x, \gamma_{\varphi}(x)\right), Z\right)\right)
$$

is a continuous semi-algebraic function of the argument $(x, \varphi) \in Y^{(s-1)}$ (cf. [BC], Proposition 2.2.8). By (5.4) and (5.5) we have $p^{*} \sigma=\varsigma$ and there exists a commutative diagram

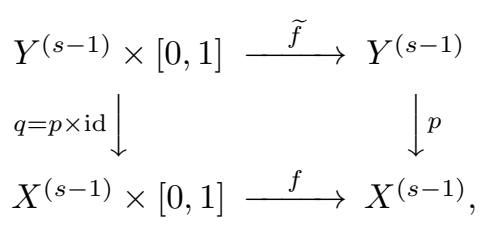

where the upper and lower horizontal arrows $\tilde{f}$ and $f$ are defined by (5.3) and (5.6), respectively, and $\tilde{f}$ is a continuous semi-algebraic map of compact spaces. From the commutative diagram we now find that the lower arrow $f$ is also continuous. Furthermore, the graph of the map $f$ is the projection of the graph of the map $\tilde{f}$, that is, $\{(a, f(a))\}=\{(q b, f(q b))\}=\{(q b, p \widetilde{f}(b))\}$. By the Tarski-Seidenberg theorem, $f$ has a semi-algebraic graph. Therefore $f$ is a continuous semi-algebraic map.

3) The restriction of $f$ to $X^{(s-1)} \times 0 \cup X^{(s)} \times[0,1]$ is the identity map, since $\left.\sigma\right|_{X^{(s)}}=0$. By (5.7), we have $d\left(f(x, 1), X^{(s)}\right)<\delta$ for all $x \in X^{(s-1)}$. Then the third assertion follows from Lemma 4.5 on $\delta$-neighbourhoods. The proposition is proved.

\section{$\S 6$. The SPACE $X_{\varepsilon}$ AND ITS RETRACTS}

6.1. The second retraction theorem. The notion of a butterfly prompts a stronger version of another of Böhm's retraction theorems (see $[\mathrm{B}$, Theorem 5.48 and Corollary 5.49) and makes it possible to trivialize its proof. We state a natural generalization of this theorem, which uses compact unions of butterflies and the standard notion of an order ideal.

Let $\mathcal{J}$ denote the upper semilattice of all $(\mathcal{A}, \mathfrak{h})$-invariant subalgebras $\mathfrak{k}, \mathfrak{h}<\mathfrak{k} \leq \mathfrak{g}$. An order ideal of $\mathcal{J}$ is defined to be any subset $\mathcal{J}$ satisfying the following condition: $\mathfrak{l} \leq \mathfrak{j}$ for $\mathfrak{l} \in \mathcal{J}, \mathfrak{j} \in \mathcal{J}$ implies $\mathfrak{l} \in \mathcal{J}$. An order ideal $\mathcal{J}$ of $\mathcal{J}$ such that $\mathfrak{g} \notin \mathcal{J}$ is called admissible if $\mathcal{J}$ and its complement $\mathcal{J} \backslash \mathcal{J}$ are compact semi-algebraic subsets of the (disconnected) manifold of vector subspaces of the algebra $\mathfrak{g}$. (For example, the so-called toral subalgebras $\mathfrak{j}$ of 
the compact Lie algebra $\mathfrak{g}$ under consideration, that is, subalgebras $\mathfrak{j} \in \mathcal{J}$ with derived subalgebras contained in $\mathfrak{h},[\mathfrak{j}, \mathfrak{j}] \leq \mathfrak{h}$, form an admissible order ideal when $[\mathfrak{g}, \mathfrak{g}] \not \leq \mathfrak{h}$.)

We fix an admissible order ideal $\mathcal{J}$ of $\mathcal{J}$ and set

$$
\begin{aligned}
& \mathbb{I}:=\{\varphi \in \Delta(\mathcal{J}) \backslash \Delta(\mathcal{J}): \varphi \backslash(\max (\varphi)) \subset \mathcal{J}\}, \\
& \mathbb{J}:=\{\varphi \in \Delta(\mathcal{J}) \backslash \Delta(\mathcal{J}): \varphi \backslash(\mathfrak{g}) \subset \mathcal{J}\}=\{\varphi \in \mathbb{I}: \mathfrak{g} \in \varphi\} .
\end{aligned}
$$

Let $\mathrm{B}[\mathcal{J}]:=\bigcup_{\mathfrak{f} \in \mathcal{J}} \mathrm{B}[\mathfrak{f}], \ldots, \mathrm{B}[\mathbb{J}]:=\bigcup_{\varphi \in \mathbb{J}} \mathrm{B}[\varphi]$. One can show that each of the sets $\mathrm{B}[\mathcal{J}], \mathrm{B}[\mathcal{J}]$, $\mathrm{B}[\mathcal{J} \backslash \mathcal{J}], \mathrm{B}[\mathbb{I}], \mathrm{B}[\mathbb{J}]$ is semi-algebraic and compact.

Theorem 6.1. The topological space $\mathrm{B}[\mathcal{J} \backslash \mathcal{J}]=\bigcup_{\mathfrak{f} \in \mathcal{J} \backslash \mathfrak{J}} \mathrm{B}[\mathfrak{f}]$ is a strong deformation retract of the complement $\mathrm{B}[\mathbb{I}] \backslash \mathrm{B}[\mathbb{J}]$.

The theorem is stated simultaneously for the rough and fine versions (that is, $\mathrm{B}=\mathrm{D}$ or $\mathrm{X}$ ) and is also valid in the semi-algebraic category.

We now explain its geometric meaning. The subspaces $\mathrm{B}[\mathrm{J}]$ and $\mathrm{B}[\mathcal{J} \backslash \mathcal{J}]$ are disjoint. The first of them is the union of rectilinear simplexes $\mathrm{B}[\varphi]=/ \varphi \backslash \mathfrak{g} /$, where $\varphi \in \mathbb{J}$. The complement of these two subspaces in $B[\mathbb{I}]$ somewhat resembles a linear congruence, since $B[\mathbb{I}] \backslash(B[\mathbb{J}] \cup B[\mathcal{J} \backslash \mathcal{J}])$ is the union of pairwise disjoint intervals of Euclidean space connecting points of the subspace $\mathrm{B}[\mathbb{J}]$ with points of the subspace $\mathrm{B}[\mathcal{J} \backslash \mathcal{J}]$ (according to a certain rule). The intervals can be simultaneously contracted to their endpoints belonging to the subspace $\mathrm{B}[\mathcal{J} \backslash \mathcal{J}]$. This produces a strong deformation retraction of $\mathrm{B}[\mathrm{I}] \backslash \mathrm{B}[\mathrm{J}]$ to $\mathrm{B}[\mathcal{J} \backslash \mathcal{J}]$.

We now elaborate on this description, and then prove Theorem 6.1. By Lemma 5.3 , for every flag $\varphi \in \mathbb{I}$ a natural embedding $\iota=\iota_{\varphi}: \mathrm{B}[\varphi] \subset Y * X$ exists, taking the butterfly $\mathrm{B}[\varphi]$ into the join of the topological spaces $X=\mathrm{B}[\mathcal{J} \backslash \mathcal{J}]$ and $Y=\mathrm{B}[\mathbb{J}]$. For every $A \in \mathrm{B}[\varphi]$, let $\iota(A)=\left(A_{1}, \kappa, A_{2}\right)$, where $A_{1} \in Y, \kappa \in[0,1], A_{2} \in X$. Then $A=\theta\left(A_{1}, \kappa, A_{2}\right)=(1-\kappa) A_{1}+\kappa A_{2}$.

Proposition 6.2. The subset $Z=\bigcup_{\varphi \in \mathbb{I}} \iota(\mathrm{B}[\varphi])$ of the join $Y * X=\mathrm{B}[\mathbb{J}] * \mathrm{~B}[\mathcal{J} \backslash \mathcal{J}]$ is defined by the following system of equations with respect to $A_{1} \in Y$ and $A_{2} \in X$ :

$$
\begin{gathered}
A_{1} A_{2}=A_{2} A_{1}=\lambda_{1} A_{2}, \quad \text { where } \lambda_{1}=\max _{|V|=1} Q\left(V, A_{1} V\right) ; \\
A_{1}\left[V, A_{2} V\right]=\lambda_{1}\left[V, A_{2} V\right] \quad \text { for every vector } V \in \mathfrak{g} .
\end{gathered}
$$

Then $Z$ is compact. The map $\theta: Y * X \ni\left(A_{1}, \kappa, A_{2}\right) \mapsto A=(1-\kappa) A_{1}+\kappa A_{2}$ defines a homeomorphism of $Z$ onto $\mathrm{B}[\mathbb{I}]=\bigcup_{\varphi \in \mathbb{I}} \mathrm{B}[\varphi]$.

(In the X-version, the second subsystem of equations follows from the first one.)

Proof. We begin by proving that $Z$ is defined by the system $(*)$. First we transform it. Strictly speaking, this is a system of homogeneous equations with respect to $(1-\kappa) A_{1}$ and $\kappa A_{2}$, which becomes trivial for $\kappa=0$ and $\kappa=1$. We fix $\kappa \in(0,1)$. From any $A_{1} \in \mathrm{B}[\mathrm{J}]$ we can uniquely reconstruct the flag subalgebra $\psi=\left(\mathfrak{g}>\mathfrak{j}_{1}>\ldots>\mathfrak{j}_{r}\right), \mathfrak{j}_{i} \in \mathcal{J}, r \geq 1$, such that $A_{1}=\sum s_{i} \bar{\chi}^{j_{i}}, \sum s_{i}=1$, and $s_{i}>0$ for all $i$ (that is, $A_{1}$ belongs to the butterfly of the 2 nd type $\mathrm{B}[\psi]$, which is a simplex, and in fact is in the interior of the simplex). Next, from $A_{2} \in \mathrm{B}[\mathcal{J} \backslash \mathcal{J}]$ we construct $\mathfrak{k}=\left\{V \in \mathfrak{g}: Q\left(V, A_{2} V^{\prime}\right)=Q\left(V,\left[V^{\prime}, A_{2} V^{\prime}\right]\right)=0 \forall V^{\prime} \in\right.$ $\mathfrak{g}\}$. It easily follows from (4.1) and (4.2) that $\mathfrak{k}$ is the largest of the subalgebras $\mathfrak{f}<\mathfrak{g}$ such that $A_{2} \in \mathrm{B}[\mathfrak{f}]$ (hence, $\mathfrak{k} \in \mathcal{J} \backslash \mathcal{J}$ ). The system $(*)$ for $\left(A_{1}, \kappa, A_{2}\right)$ is equivalent to the condition $\mathfrak{k}>\mathfrak{j}_{1}$.

Let $\left(A_{1}, \kappa, A_{2}\right)=\iota_{\varphi}(A) \in Z$ for some flag $\varphi=\left(\mathfrak{f}_{0}>\mathfrak{f}_{1}>\ldots>\mathfrak{f}_{m}\right) \in \mathbb{I}$. Then $A_{2} \in$ $\mathrm{B}\left[\mathfrak{f}_{0}\right]$ and $\mathfrak{f}_{0} \leq \mathfrak{k}$. Furthermore, $\mathrm{B}[\psi]$ is a face of the simplex $\mathrm{B}\left[\mathfrak{g}>\mathfrak{f}_{1}>\ldots>\mathfrak{f}_{m}\right] \ni A_{1}$, and $\mathfrak{j}_{1} \leq \mathfrak{f}_{1}$. Consequently, $\mathfrak{j}_{1}<\mathfrak{k}$. Conversely, let $\mathfrak{k}>\mathfrak{j}_{1}$. Then there exists a flag $\varphi_{0}=\left(\mathfrak{k}>\mathfrak{j}_{1}>\ldots>\mathfrak{j}_{r}\right) \in \mathbb{I}$; clearly, $A \in \mathrm{B}\left[\varphi_{0}\right]$ and $\left(A_{1}, \kappa, A_{2}\right)=\iota_{\varphi_{0}}(A) \in Z$. 
We now prove the other assertions. Obviously, $(1-\kappa) \lambda_{1}$ is a continuous semi-algebraic function of the operator $(1-\kappa) A_{1}$ (this is its largest eigenvalue). Hence the system $(*)$ is continuous. Then as $X$ and $Y$ are compact so is $Z$. We now verify that $\left.\theta\right|_{Z}$ is invertible. For any flags $\varphi, \psi \in \mathbb{I}$, if $\psi \geq \varphi$ it follows that $\iota_{\psi}=\left.\iota_{\varphi}\right|_{\mathrm{B}[\psi]}$. Then by formula (5.2) for the intersection of butterflies, $\left.\iota_{\varphi_{1}}\right|_{\mathrm{B}\left[\varphi_{1}\right] \cap \mathrm{B}\left[\varphi_{2}\right]}=\iota_{\varphi_{1} \varphi_{2}}$ and the map $\iota=\bigcup_{\varphi \in \mathbb{I}} \iota_{\varphi}: \mathrm{B}[\mathbb{I}] \rightarrow Z$ exists. Hence $\left.\theta\right|_{Z}$ is invertible and is a homeomorphism onto $\mathrm{B}[\mathbb{I}]$.

Proposition 6.2 still makes sense in the semi-algebraic category. This proposition also makes it possible to introduce a natural continuous semi-algebraic function $\kappa: \mathrm{B}[\mathbb{I}] \rightarrow$ $[0,1]$ such that $\mathrm{B}[\mathbb{J}]=\{A: \kappa(A)=0\}$ and $\mathrm{B}[\mathcal{J} \backslash \mathcal{J}]=\{A: \kappa(A)=1\}$. Hence we obviously obtain the retraction of Theorem 6.1. Furthermore, the following holds.

Proposition 6.3. Suppose that $0<\varepsilon<1$ and the lengths of the flags in $\Delta(\mathcal{J})$ are bounded above by some number $L$. Then for every operator $A \in \mathrm{B}[\mathbb{I}]$ satisfying the condition

$$
\kappa(A)<\varepsilon^{L},
$$

there exist a flag of subalgebras $\varphi=(\mathfrak{f}(1)>\ldots>\mathfrak{f}(m)) \in \Delta(\mathcal{J})$ (of length $m>0$ ) and an operator $B \in \mathrm{B}[\mathfrak{f}(1)]$ such that the Euclidean distance from $B$ to $\bar{\chi}^{\mathfrak{f}(1)}$ is less than $\varepsilon,\left|B-\chi^{f(1)}\right|<\varepsilon$, and $A$ is contained in the convex hull $S$ of the set of operators $\left\{B, \bar{\chi}^{f(s)}, 2 \leq s \leq m\right\}$. (Clearly, $S$ is a simplex.)

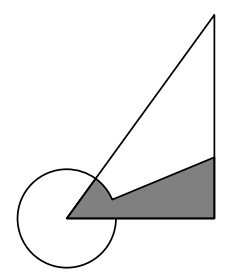

Figure 3. Towards the proof of Proposition 6.3.

Proof. Let $A \in \mathrm{B}[\varphi]$ for some flag $\varphi=\left(\mathfrak{f}_{0}>\ldots>\mathfrak{f}_{r-1}\right) \in \mathbb{I}$. If $\kappa(A)=0$, then $A$ is contained in the simplex spanned by all the $\bar{\chi}^{f_{i}}, i \in\{1, \ldots, r-1\}$, and the proposition holds. Suppose that $0<\kappa(A)<\varepsilon^{L}$. Then $\mathfrak{f}_{0} \neq \mathfrak{g}$ (otherwise, $\kappa=0$ ) and $r>1$ (otherwise, $\kappa=1)$. We can introduce a sequence of numbers $0<a_{0} \leq a_{1} \leq \ldots \leq a_{r-1}=1$ and the sequence of symmetric operators $B_{i} \in \mathrm{B}\left[\mathfrak{f}_{i}\right], i=0, \ldots, r-1$, given by $B_{i}=$ $a_{i}^{-1}\left(a_{0} B_{0}+\sum_{j=1}^{i}\left(a_{j}-a_{j-1}\right) \bar{\chi}^{\mathfrak{f}_{j}}\right)$, and the operator $A$ can be expressed simultaneously in $r$ ways in terms of them:

$$
A=a_{i} B_{i}+\sum_{j=i+1}^{r-1}\left(a_{j}-a_{j-1}\right) \bar{\chi}^{\mathfrak{f}_{j}}, \quad i=0, \ldots, r-1 .
$$

Using the orthogonality of the set $\left\{B_{0}-\bar{\chi}^{f_{0}}, \bar{\chi}^{f_{j}}-\bar{\chi}^{f_{j+1}}, j \geq 0\right\}$ and the strict inequality $\left|B_{0}-\bar{\chi}^{i}\right|<1$, we obtain

$$
\left|B_{i}-\bar{\chi}^{f_{i}}\right|^{2}=a_{i}^{-2}\left|a_{0}\left(B_{0}-\bar{\chi}^{f_{0}}\right)+\sum_{j=0}^{i-1} a_{j}\left(\bar{\chi}^{f_{j}}-\bar{\chi}^{f_{j+1}}\right)\right|^{2}<\left(a_{i-1} / a_{i}\right)^{2}
$$

(for every $i$ ), so that

$$
\prod_{i=1}^{r-1}\left|B_{i}-\bar{\chi}^{\mathfrak{f}_{i}}\right|<\kappa(A)=a_{0} / a_{r-1}
$$

Hence $\left|B_{i}-\bar{\chi}^{f_{i}}\right|<\varepsilon^{L /(r-1)} \leq \varepsilon$ for at least one $i \in\{1, \ldots, r-1\}$. 
6.2. A suitable extension $X_{\varepsilon}$ of the space of non-toral directions. We now go on to look at the definition and conclusions based on Theorems 5.1, 5.2. and 6.1 Suppose that $[\mathfrak{g}, \mathfrak{g}] \not \leq \mathfrak{h}$. Then, as we already mentioned, the toral subalgebras $\mathfrak{j} \in \mathcal{J}$ form an admissible order ideal $\mathcal{\partial}$. In accordance with [B], we call

$$
B[\mathcal{J} \backslash \mathcal{J}]=\bigcup_{\mathfrak{f} \in \mathcal{J} \backslash \mathcal{J}} B[\mathfrak{f}]
$$

the space of non-toral directions. As above, $\mathrm{B}=\mathrm{D}$ or $\mathrm{X}$ (the definition in $\mathrm{B}$ only covers the case $\mathrm{B}=\mathrm{X})$.

We turn to the definition of the extended space of non-toral directions $X_{\varepsilon}$, which can be used instead of the one introduced in [B], Theorem 5.48. The extended space admitting a strong deformation retraction first to $\mathrm{B}[\mathcal{J} \backslash \mathcal{J}]$, and then to the polyhedron $/ \mathcal{K}^{\#} /$, plays an important role in Böhm's paper (as can be seen in the proof of the main theorem in $[\mathrm{B}, \S 8)$. The original construction of the extension and its retraction to the space of toral directions $\mathrm{B}[\mathcal{J} \backslash \mathcal{J}]$ proceeds by induction and is rather involved. In reality, as will be seen in the proof of Theorem 6.1, an extension with the necessary property (described in [B], Corollary 5.49) and a retraction can be obtained in one step.

By Theorem 6.1 the topological space

$$
\mathrm{B}[\mathbb{I}] \backslash \mathrm{B}[\mathbb{J}]=\bigcup_{\varphi \in \mathbb{I}} \mathrm{B}[\varphi] \backslash \bigcup_{\mathfrak{g} \in \varphi \in \mathbb{I}} \mathrm{B}[\varphi]
$$

contains a compact subspace $X_{\varepsilon}$ contractible over itself to $\mathrm{B}[\mathcal{J} \backslash \mathcal{J}]$. Suppose that $\mathrm{B}[\mathbb{I}] \backslash X_{\varepsilon}$ is contained in a sufficiently small neighbourhood of the compact subspace $\mathrm{B}[\mathbb{\mathbb { J }}]$; this can certainly be achieved. Then $X_{\varepsilon}$ satisfies the conditions in Corollary 5.49 in [B]. For this reason, $X_{\varepsilon}$ can be used instead of the extension introduced in $[\mathrm{B}$, Theorem 5.48. We call such a subspace $X_{\varepsilon}$ a suitable extension of the space of non-toral directions.

It follows from Proposition 6.3 that for every sufficiently small $\varepsilon>0$

$$
X_{\varepsilon}=\left\{A \in \mathrm{B}[\mathbb{I}]: \kappa(A) \geq \varepsilon^{L}\right\}
$$

is a suitable extension.

Corollary 6.4. We fix $\varepsilon \in(0,1)$. The space $X_{\varepsilon}$ constructed above is compact, semialgebraic, and the space of non-toral directions $X=\mathrm{B}[\mathcal{J} \backslash \mathcal{J}]$ is a semi-algebraic strong deformation retract of it. Therefore, $X_{\varepsilon}$ satisfies the conditions listed in $[\mathrm{B}]$, Theorem 5.48 .

For every $A \in \mathrm{W}$, let $\varphi_{A}$ denote the largest of the flags $\varphi \in \Delta(\mathcal{J})$ such that $A \in \mathrm{B}[\varphi]$. Obviously, $\varphi_{A}$ exists and $\mathrm{B}\left[\varphi_{A}\right]$ is the smallest butterfly containing A.

Let $Z=\left\{A: \varphi_{A} \in \Delta(\mathcal{J})\right.$, that is, $\varphi_{A}$ is a flag of toral subalgebras $\}$, and let $Y_{\varepsilon}$ be the union of the simplexes $S$ in Proposition 6.3 above. Then

$$
\mathrm{W}=X_{\varepsilon} \cup Y_{\varepsilon} \cup Z \text {. }
$$

It follows from (6.1) and (6.2) that $X_{\varepsilon}$ is a suitable extension of the space of non-toral directions for $\varepsilon \leq \frac{1}{2 n(n-1)}$, where $n=\operatorname{dim}(G / H)$.

Corollary 6.4 concerns the fine version, $\mathrm{B}=\mathrm{X}$. With natural changes, it is also true for $\mathrm{B}=\mathrm{D}$; the first assertion does not change.

Proof. We will now prove the last assertion. If $\varepsilon \leq \frac{1}{2 n(n-1)}$, where $n=\operatorname{dim}(G / H)$, then the complement $\mathrm{W} \backslash X_{\varepsilon}$ is covered by the sets $Y$ and $Z$ defined in $[\mathrm{B}$, Corollary 5.49 , by formulae (5.50) and (5.51), since $Y \supset Y_{\varepsilon}$ and $Z$ occurs in (6.2). In this case, $X_{\varepsilon}$ in (6.1) is a suitable extension of the space of non-toral directions 10

\footnotetext{
${ }^{10}$ Recall that another model $W^{\Sigma} \subset \Sigma$ of the topological space $\mathrm{W} \subset \mathrm{S}$ was constructed in $[\mathrm{B}]$ (see $\S[$ ). When passing to $\Sigma$, the radius $\varepsilon$ in Proposition 6.3 is replaced by $\varepsilon^{\dagger} \leq \varepsilon\left|\chi^{(1)}-\bar{\chi}^{h}\right|^{-1} \leq \varepsilon \sqrt{n(n-1)}$.
} 
We pass to upper semilattices $\mathcal{K} \subset \mathcal{J}$. By definition, each of these semilattices $\mathcal{K}$, together with any subalgebras $\mathfrak{k}$ and $\mathfrak{l}$, also contains the smallest subalgebra containing them $\mathfrak{f}=\sup (\mathfrak{k}, \mathfrak{l})$.

A non-toral subalgebra $\mathfrak{i}, \mathfrak{i} \in \mathcal{J} \backslash \mathcal{J}$, is called a minimal element of the filter $\mathcal{J} \backslash \mathcal{J}$ if $\mathcal{J} \backslash \mathcal{J}$ does not contain any smaller subalgebra, that is, $\mathfrak{j} \in \mathcal{J}, \mathfrak{j}<\mathfrak{i}$ implies $\mathfrak{j} \in \mathcal{J}=$ $\{\mathfrak{j} \in \mathcal{J}:[\mathfrak{j}, \mathfrak{j}] \leq \mathfrak{h}\}$.

Let $\mathcal{K}^{\text {min }}$ denote the smallest upper semilattice 11 in $\mathcal{J} \backslash \mathcal{J}$ containing $\mathfrak{g}$ and all the minimal elements in $\mathcal{J} \backslash \mathcal{J}$. Then we have the equation

$$
\mathrm{B}[\mathcal{J} \backslash \mathcal{J}]=\mathrm{B}\left[\mathcal{K}^{\text {min }}\right]:=\bigcup_{\mathfrak{k} \in \mathcal{K}^{\min }} \mathrm{B}[\mathfrak{k}] .
$$

We now consider a finite upper semilattice $\mathcal{K} \subset \mathcal{J}$. Earlier the polyhedron corresponding to $\mathcal{K}^{\#}=\{\mathfrak{k} \in \mathcal{K}: \mathfrak{k} \neq \mathfrak{g}\}$ was denoted by $/ \mathcal{K}^{\#} /$ and called admissible.

Theorems 5.1 and 5.2 and equation (6.3) imply the following.

Corollary 6.5. Let $\mathcal{K}$ be a finite upper semilattice contained (not necessarily strictly) between $\mathcal{K}^{\text {min }}$ and the semilattice $\mathcal{J} \backslash \mathcal{J}$ of all $(\mathcal{A}, \mathfrak{h})$-invariant non-toral subalgebras. Then the polyhedron $/ \mathcal{K}^{\#} /$ is a strong deformation retract of the space $X_{\varepsilon}$.

The simplicial complex $\Delta^{\text {min }}:=\Delta\left(\left(\mathcal{K}^{\text {min }}\right)^{\#}\right)$ connected with $G / H$ and its geometric realization $/\left(\mathcal{K}^{\mathrm{min}}\right)^{\# /}$ were considered in $[\mathrm{B}$. Note that its vertices correspond to algebraic subalgebras of the algebra $\mathfrak{g}$ (see the footnote). Furthermore, in $\S 7$ of $[\mathrm{B}$ it was shown that the following simple additional condition on the group $\mathcal{A}: \operatorname{Ad}(T) \subset \mathcal{A} \subset$ $\operatorname{Aut}(\mathfrak{g}, \mathfrak{h})$, where $T$ is a maximal torus of the group $\operatorname{Norm}_{G}(\mathfrak{h})$, implies that $\mathcal{K}^{\text {min }}$ is finite.

6.3. Retraction to $/ \mathcal{K}^{\#} /$. The case of a compact semilattice $\mathcal{K}$. Suppose that, as in Proposition 5.5, $\mathcal{K} \subset \mathcal{J}$ is a compact semi-algebraic upper semilattice of $(\mathcal{A}, \mathfrak{h})$ invariant subalgebras $\mathfrak{k}, \mathfrak{h}<\mathfrak{k} \leq \mathfrak{g}$, no longer necessarily finite, such that $\mathcal{K} \ni \mathfrak{g}$, and let $\mathcal{K}^{\#}:=\mathcal{K} \backslash(\mathfrak{g})$. We again denote by $/ \mathcal{K}^{\#} /$ the union of all simplexes of the form

$$
|\varphi|:=\text { Convex hull }\left\{\bar{\chi}^{\mathfrak{f}_{i}}: i=1, \ldots, r\right\} \subset \mathfrak{g l}(\mathfrak{g}),
$$

where $\varphi=\left(\mathfrak{f}_{1}>\ldots>\mathfrak{f}_{r}\right)$ is a flag of subalgebras in $\mathcal{K}^{\#}$ of length $r \geq 1$. Then $/ \mathcal{K}^{\#} / \subset \mathfrak{g l}(\mathfrak{g})$ is a compact semi-algebraic subset by Proposition 5.5 .

Theorem 6.6. The compact set $/ \mathcal{K}^{\#} /=\bigcup_{\varphi \in \Delta\left(\mathcal{K}^{\#}\right)} / \varphi /$ is a strong deformation retract of the following compact sets:

$$
\mathrm{B}[\mathcal{K}]:=\bigcup_{\mathfrak{k} \in \mathcal{K}^{\#}} \mathrm{~B}[\mathfrak{k}] \quad \text { in the general case }
$$

$$
X_{\varepsilon} \text { and } X_{1}=\mathrm{B}[\mathcal{J} \backslash \mathcal{J}] \text { for }[\mathfrak{g}, \mathfrak{g}] \not \subset \mathfrak{h} \text { and } \mathcal{K}^{\text {min }} \subset \mathcal{K} \subset \mathcal{J} \backslash \mathcal{J} .
$$

Here, $X_{\varepsilon}=\left\{A \in \mathrm{B}[\mathbb{I}]: \kappa(A) \geq \varepsilon^{L}\right\}$ for every $\varepsilon \in(0,1)$ and $X_{1} \subset X_{\varepsilon}$ is by definition the space of non-toral directions.

By $\left[\mathrm{B}\right.$, we can take the radius $\varepsilon^{\dagger} \leq \varepsilon_{G / H}=\frac{1}{2}\left|c_{G / H}\right|$, where

$$
c_{G / H}=\max _{v \in \Sigma} \min _{X \in \mathfrak{g},|X|=1} Q(v X, X)=-\min _{v \in \Sigma} \max _{X \in \mathfrak{g},|X|=1} Q(v X, X) \leq \frac{-1}{\sqrt{n(n-1)}}
$$

(cf. B], $\S \S 4.2,5.4,5.7)$. Then $\varepsilon^{\dagger} \leq \frac{1}{2 \sqrt{n(n-1)}} \leq \varepsilon_{G / H}$ if $\varepsilon \leq \frac{1}{2 n(n-1)}$.

${ }^{11}$ Every minimal element $\mathfrak{l} \in \mathcal{J} \backslash \mathfrak{d}$ is contained in the upper semilattice

$$
\mathcal{L}:=\{\mathfrak{l} \in \mathfrak{J}:[\mathfrak{l}, \mathfrak{l}]+\mathfrak{h}=\mathfrak{l}\} .
$$

We point out that (if we start from a compact homogeneous Riemannian space $G / H$ ) every subalgebra $\mathfrak{l} \in \mathcal{L}$ corresponds to a compact subgroup of the group $G$. 
The theorem is stated simultaneously for the fine and rough versions, that is, for $\mathrm{B}=\mathrm{X}$ and $\mathrm{D}$, respectively, and is valid in the semi-algebraic category.

Proof. Proposition [5.5] asserts the existence of a sequence $X^{(0)} \rightarrow \ldots \rightarrow X^{(m)}$ of semialgebraic strong deformation retractions of compact spaces, where $X^{(m)}=/ \mathcal{K}^{\#} /$ and $X^{(0)}=\mathrm{B}[\mathcal{K}]$. This proves the first part of the theorem.

Now suppose that the semilattice $\mathcal{K}$ is contained (possible, not strictly) between the semilattices of the non-toral subalgebras $\mathcal{K}^{\text {min }}$ and $\mathcal{J} \backslash \mathcal{J}$. Then $\mathrm{B}\left[\mathcal{K}^{\mathrm{min}}\right]=\mathrm{B}[\mathcal{K}]=\mathrm{B}[\mathcal{J} \backslash \mathcal{J}]$. But this is a semi-algebraic strong deformation retract of the space $X_{\varepsilon}$ (Corollary 6.4), and the second part follows from the first.

\section{$\S$ 7. Supplement. The family of toral subalgebras}

In this supplement we prove that the toral subalgebras form a compact open subset of the set $\mathcal{J}$ of all $\mathcal{A}$-invariant subalgebras $\mathfrak{l}$ of the Lie algebra $\mathfrak{g}$ properly containing an $\mathcal{A}$-invariant subalgebra $\mathfrak{k}, \mathfrak{k}<\mathfrak{l} \leq \mathfrak{g}$.

Assertion. Suppose that $[\mathfrak{g}, \mathfrak{g}] \not \subset \mathfrak{k}$, and let $\mathcal{J}=\{\mathfrak{j} \in \mathcal{J}:[\mathfrak{j}, \mathfrak{j}] \subset \mathfrak{k}\}$, that is, $\mathcal{J}$ is the subset of toral subalgebras with respect to $\mathfrak{k}$. Then $\mathcal{J}$ and $\mathcal{J} \backslash \mathcal{J}$ are compact. Moreover, the function $\mathfrak{l} \in \mathcal{J} \mapsto \operatorname{dim}([\mathfrak{l}, \mathfrak{l}])$ is continuous.

Proof. With every subalgebra $\mathfrak{l} \subset \mathfrak{g}$, we associate a rational number $c_{1}(\mathfrak{l}) \in \mathbb{Q}$ by fixing an embedding $\rho: G \subset S O(N)$ (where $G$ is compact) and setting $F(X, X)=\operatorname{trace}\left(d \rho(X)^{2}\right)$, $X \in \mathfrak{g}$. Let $\left\{Z_{i}\right\}$ be any $F$-orthonormal basis in $\mathfrak{l}$, and let $C=-\sum_{i=1}^{\operatorname{dim}(\mathfrak{l})}\left(\operatorname{ad}_{\mathfrak{l}} Z_{i}\right)^{2} \in$ $\operatorname{End}(\mathfrak{l})$. Then the sequence of numbers $c_{1}(\mathfrak{l})=\operatorname{trace}(C), \ldots, c_{r}(\mathfrak{l})=\operatorname{trace}\left(\bigwedge^{r} C\right), \ldots$ depends only on $\mathfrak{l}$. We have $c_{r}(\mathfrak{l})=c_{r}\left(\mathfrak{l}^{\prime}\right)$, where $\mathfrak{l}^{\prime}=[\mathfrak{l}, \mathfrak{l}]$ is the derived subalgebra of the algebra $\mathfrak{l}$, in other words, its largest semisimple subalgebra. Therefore, $c_{r}(\mathfrak{l}) \in \mathbb{Q}$. Furthermore, $\operatorname{dim}\left(\mathfrak{l}^{\prime}\right)=\max \left\{r: c_{r}(\mathfrak{l})>0\right\}$. Let $A$ be a linear connected component of the compact algebraic set of $p$-dimensional subalgebras $\mathfrak{l}, \mathfrak{l}>\mathfrak{k}$. Then $c_{r}: A \rightarrow \mathbb{Q}$ is a continuous and therefore constant function, $r=1,2, \ldots$. By complementing a basis of a subalgebra $\mathfrak{l} \in A$ with a basis of the subalgebra $\mathfrak{k}^{\prime}$, we obtain $c_{r}(\mathfrak{l})>c_{r}\left(\mathfrak{k}^{\prime}\right)$ for $\mathfrak{l}^{\prime}>\mathfrak{k}^{\prime}$ and $c_{r}(\mathfrak{l})=c_{r}\left(\mathfrak{k}^{\prime}\right)$ for $\mathfrak{l}^{\prime}=\mathfrak{k}^{\prime}$. For $r=1$ this implies the first assertion.

The Assertion was used in $\S \S 6.2$ and 6.3 . The proof of Lemma 5.42 in $[\mathrm{B}$ also uses the fact that $\mathcal{J} \backslash \mathcal{J}$ and $\mathcal{J}$ are compact.

\section{REFERENCES}

[Be] A. L. Besse, Einstein manifolds, Ergeb. Math. und ihrer Grenzgeb. (3), vol. 10, Springer-Verlag, Berlin, 1987. MR867684 (88f:53087)

[B] C. Böhm, Homogeneous Einstein metrics and simplicial complexes, J. Differential Geom. 67 (2004), no. 1, 79-165. MR2153482 (2006m:53065)

[BK] C. Böhm and M. M. Kerr, Low-dimensional homogeneous Einstein manifolds, Trans. Amer. Math. Soc. 358 (2006), no. 4, 1455-1468. MR2186982 (2006g:53056)

[BWZ] C. Böhm, M. Wang, and W. Ziller, A variational approach for compact homogeneous Einstein manifolds, Geom. Funct. Anal. 14 (2004), no. 4, 681-733. MR2084976 (2005g:53074)

[BC] J. Bochnak, M. Coste, and M.-F. Roy, Real Algebraic Geometry, Ergeb. Math. und ihrer Grenzgeb. (3), vol. 36, Springer-Verlag, Berlin, 1998. MR1659509 (2000a:14067)

[GLP] G. W. Gibbons, H. Lu, and C. N. Pope, Einstein Metrics on Group Manifolds and Cosets, Preprint arXiv:0903.2493 MR2776894 (2012d:53138)

[J] G. Jensen, The scalar curvature of left-invariant Riemannian metrics, Indiana Univ. Math. J. 20 (1971), no. 12, 1125-1144. MR0289726 (44:6914) 
[WZ1] M. Wang and W. Ziller, On normal homogeneous Einstein manifolds, Ann. Sci. École Norm. Sup. (4), 18 (1985), no. 4, 563-633. MR839687 (87k:53113)

[WZ2] M. Wang and W. Ziller, Existence and nonexistence of homogeneous Einstein metrics, Invent. Math. 84 (1986), no. 1, 177-194. MR830044 (87e:53081)

Scientific Research Institute of System Studies of the Russian Academy of Sciences

E-mail address: mmgraev@gmail.com

Translated by E. KHUKHRO

Originally published in Russian 